\title{
Equivalence of the 11D pure spinor and Brink-Schwarz-like superparticle cohomologies
}

\author{
Max Guillen* \\ Instituto de Física Teórica, UNESP-Universidade Estadual Paulista Rua Doutor Bento Teobaldo Ferraz. \\ 271, Bloco II, Barra-Funda, São Paulo 01140-070, São Paulo, Brazil
}

(Received 24 January 2018; published 7 March 2018)

\begin{abstract}
The $D=11$ pure spinor formulation of the superparticle allows a simple realization of covariant quantization, unlike the $D=11$ Brink-Schwarz-like superparticle. We explicitly show the equivalence between the cohomologies of these two models in the context of two different group decompositions: $S O(10,1) \rightarrow S O(1,1) \times S O(9)$ and $S O(10,1) \rightarrow S O(3,1) \times S O(7)$. We also carry out a light-cone analysis of the pure spinor cohomology and show that it correctly reproduces the $S O(9)$ equations of motion for $D=11$ linearized supergravity.
\end{abstract}

DOI: 10.1103/PhysRevD.97.066002

\section{INTRODUCTION}

It is well known that the $D=10$ Brink-Schwarz formulation of the superparticle possesses first- and secondclass constraints which cannot be separated out in a manifestly covariant way. If the physical spectrum is our main concern, we can always go to the light-cone gauge and follow Dirac's prescription to show that the physical spectrum consists of an $S O(8)$ vector and spinor, which satisfy the $D=10$ linearized super-Yang-Mills (SYM) equations of motion [1]. However, cone gauge breaks the manifest covariance of the theory.

It is interesting and useful to look for covariant descriptions which manifestly preserve as many symmetries as possible. One candidate that addresses this point is the pure spinor version of the $D=10$ BrinkSchwarz superparticle, known as the $D=10$ pure spinor superparticle $[2,3]$. This description preserves supersymmetry and Lorentz symmetry in a manifestly covariant way. The spectrum is defined as the cohomology of the Becchi-Rouet-Stora-Tyutin (BRST) operator defined by $Q=\lambda^{\mu} d_{\mu}$, where $\lambda^{\mu}$ is a $D=10$ pure spinor and the $d_{\mu}$ are the fermionic constraints of the $D=10$ BrinkSchwarz superparticle. There are two ways to see that the pure spinor formulation indeed describes $D=10$ linearized super-Yang-Mills. The first one is by looking at the $Q$-cohomology of the $D=10$ pure spinor superparticle and realizing that the elements in this cohomology

\footnotetext{
*luismax@ift.unesp.br
}

Published by the American Physical Society under the terms of the Creative Commons Attribution 4.0 International license. Further distribution of this work must maintain attribution to the author(s) and the published article's title, journal citation, and DOI. Funded by SCOAP ${ }^{3}$. describe the Batalin-Vilkovisky (BV) version of $D=10$ (Abelian) super-Yang-Mills [2]. The second one is by showing that the cohomologies corresponding to the $D=10$ Brink-Schwarz superparticle and the $D=10$ pure spinor superparticle are identical [4].

As explained in Refs. [2-5], the $D=10$ SYM physical fields are found in the ghost-number 1 vertex operator $V=\lambda^{\mu} A_{\mu}$, after imposing on it the pure spinor physical state condition. The light-cone analysis of this cohomology reproduces the $S O(8)$ superfield $A_{a}$ satisfying the SYM equations of motion in $D=8$ superspace [6].

In $D=11$, the story is similar. The $D=11$ BrinkSchwarz-like superparticle [7] possesses first-class and second-class constraints which do not allow a manifestly covariant quantization of the theory. However, it is possible to quantize the theory in the light-cone gauge, and it can be shown that the spectrum is described by an $S O(9)$ traceless symmetric tensor, an $S O(9) \Gamma$-traceless vector-spinor, and an $S O(9)$ 3-form which describe $D=11$ linearized supergravity. As before, this theory is no longer manifestly Lorentz covariant.

As in the $D=10$ case, Berkovits formulated the so-called $D=11$ pure spinor superparticle [5]. The physical states of this pure spinor version are defined as elements in the cohomology of the BRST operator $Q=\Lambda^{\alpha} D_{\alpha}$, where $\Lambda^{\alpha}$ is a $D=11$ pure spinor and $D_{\alpha}$ are the fermionic constraints of the $D=11$ BrinkSchwarz-like superparticle. The elements of this $Q$-cohomology describe the BV version of $D=11$ linearized supergravity [5]. Unlike the $D=10$ case, there is not explicit proof of the equivalence between the cohomologies of the $D=11$ BrinkSchwarz-like superparticle and the $D=11$ pure spinor 
superparticle. ${ }^{1}$ In this work, we will demonstrate the equivalence of these two cohomologies by using two different group decompositions. ${ }^{2}$

As explained in Ref. [5], the $D=11$ supergravity physical fields are found in the ghost-number 3 vertex operator $V=\Lambda^{\alpha} \Lambda^{\beta} \Lambda^{\delta} C_{\alpha \beta \delta}$, after imposing the pure spinor physical state condition. The light-cone analysis of this cohomology will be described by the $S O(9)$ superfields $g_{j k}$, $\tilde{\psi}_{A}^{j}$, and $C_{j k l}$, which satisfy a set of equations of motion in $D=9$ superspace that match the linearized supergravity light-cone equations of motion [7].

The paper is organized as follows. In Sec. II, we review the $D=11$ Brink-Schwarz-like superparticle. In Sec. III, we present the $D=11$ pure spinor superparticle and show the equivalence between the cohomologies of this theory and the previous one by decomposing $D=11$ objects into their $S O(1,1) \times S O(9)$ and $S O(3,1) \times S O(7)$ components. In Sec. IV, we study the light-cone pure spinor cohomology and show that it is described by the usual $S O$ (9) irreducible representations that describe $D=11$ supergravity and satisfy linearized equations of motion in $D=9$ superspace.

\section{REVIEW OF THE $D=11$ BRINK-SCHWARZ-LIKE SUPERPARTICLE}

The $D=11$ Brink-Schwarz-like superparticle is defined by the action $[5,7]$

$$
S=\int d \tau\left(P^{m} \Pi_{m}+e P^{m} P_{m}\right),
$$

where $\Pi_{m}=\dot{X}_{m}-\dot{\Theta}^{\alpha}\left(\Gamma_{m}\right)_{\alpha \beta} \Theta^{\beta}$ and $\Theta^{\alpha}$ is a Majorana spinor. Let us now fix conventions. We will denote $S O(10,1)$ vector indices by $m, n, p, \ldots$, and spinor indices by $\alpha, \beta, \ldots(m=0, \ldots, 10$ and $\alpha=1, \ldots, 32)$. The $D=11$ gamma matrices $\Gamma^{m}$ are $32 \times 32$ symmetric matrices which satisfy $\Gamma_{\alpha \beta}^{m} \Gamma^{n \beta \gamma}+\Gamma_{\alpha \beta}^{n} \Gamma^{m \beta \gamma}=2 \eta^{m n} \delta_{\alpha}^{\gamma}$ and $\eta_{m n} \Gamma_{(\alpha \beta}^{m} \Gamma_{\gamma \delta)}^{n p}=0$. In contrast to the $D=10$ case, in $D=11$, there exists an antisymmetric metric tensor $C_{\alpha \beta}$ [and its inverse $\left(C^{-1}\right)^{\alpha \beta}$ ], which will allow us to lower (and raise) indices (for instance $\Gamma^{m \alpha \beta}=C^{\alpha \delta} \Gamma_{\delta}^{m \beta}$, etc). We also note that any $D=$ 11 antisymmetric bispinor can be decomposed into a scalar, 3-form, and 4-form as $f^{[\alpha \beta]}=C^{\alpha \beta} f+\left(\Gamma_{m n p}\right)^{\alpha \beta} f^{m n p}+$ $\left(\Gamma_{\text {mnpq }}\right)^{\alpha \beta} f^{m n p q}$ and that any $D=11$ symmetric bispinor

\footnotetext{
${ }^{1}$ There is a brief discussion of this point in Ref. [8], which suggests following the same ideas developed in the $D=10$ case. We will elaborate on the ideas mentioned there and give another way to parametrize $D=11$ pure spinors.

${ }^{2}$ In Refs. [9,10], I. Bandos relates these two models by using the Lorentz harmonics approach. We will address the problem in a different way, by focusing on the $D=11$ light-cone BrinkSchwarz-like superparticle.
}

can be written in terms of a 1-form, 2-form, and 5-form as $g^{(\alpha \beta)}=\Gamma_{m}^{\alpha \beta} g^{m}+\left(\Gamma_{m n}\right)^{\alpha \beta} g^{m n}+\left(\Gamma_{m n p q r}\right)^{\alpha \beta} g^{m n p q r}$.

The action (1) is invariant under reparametrizations, supersymmetry (SUSY) transformations, and $\kappa$ transformations, which are defined by the following equations:

$$
\begin{aligned}
& \text { reparametrizations } \rightarrow d \tau^{\prime}=\frac{d \tau^{\prime}}{d \tau} d \tau, \\
& e^{\prime}\left(\tau^{\prime}\right)=\frac{d \tau}{d \tau^{\prime}} e(\tau)
\end{aligned}
$$

SUSY transformations $\rightarrow \delta \Theta^{\alpha}=\epsilon^{\alpha}, \quad \delta X^{m}=\Theta \Gamma^{m} \epsilon$,

$$
\delta P_{m}=\delta e=0
$$

$\kappa$ transformations $\rightarrow \delta \Theta^{\alpha}=i P^{m}\left(\Gamma_{m} \kappa\right)^{\alpha}$,

$$
\begin{aligned}
\delta P_{m} & =0, \\
\delta X^{m} & =-\Theta \Gamma^{m} \delta \Theta^{\beta}, \\
\delta e & =2 i \dot{\Theta}^{\beta} \kappa_{\beta} .
\end{aligned}
$$

The conjugate momentum to $\Theta^{\alpha}$ is

$$
P_{\alpha}=\frac{\partial L}{\partial \dot{\Theta}^{\alpha}}=-\Gamma_{\alpha \beta}^{m} \Theta^{\beta} P_{m}
$$

Therefore, this system possesses constraints,

$$
D_{\alpha}=P_{\alpha}+\Gamma_{\alpha \beta}^{m} \Theta^{\beta} P_{m}
$$

and considering that $\left\{\Theta^{\alpha}, P_{\beta}\right\}_{P . B}=i \delta_{\beta}^{\alpha}$, we get the constraint algebra

$$
\left\{D_{\alpha}, D_{\beta}\right\}=2 i\left(\Gamma^{m}\right)_{\alpha \beta} P_{m},
$$

where $\{\cdot, \cdot\}$ denotes a Poisson bracket. One can show that $K^{\alpha}=P^{m} \Gamma_{m}^{\alpha \beta} D_{\beta}$ are the first-class constraints that generate the $\kappa$-symmetry. From (4), we realize that we have 16 firstclass constraints and 16 second-class constraints, and there is no simple way to covariantly separate them out. However, the physical spectrum can be easily found by using the semi-light-cone gauge, which is defined by

$$
\begin{array}{ll}
X^{+}=\frac{1}{\sqrt{2}}\left(X^{0}+X^{9}\right), & \Gamma^{+}=\frac{1}{\sqrt{2}}\left(\Gamma^{0}+\Gamma^{9}\right) \\
X^{-}=\frac{1}{\sqrt{2}}\left(X^{0}-X^{9}\right), & \Gamma^{-}=\frac{1}{\sqrt{2}}\left(\Gamma^{0}-\Gamma^{9}\right) .
\end{array}
$$

In these light-cone coordinates, one can use the $\kappa$ transformation to choose a gauge where $\left(\Gamma^{+} \Theta\right)_{\alpha}=0^{3}$ With this choice, we can rewrite the action as follows,

\footnotetext{
${ }^{3}$ An easy way to see this is to choose a frame where $P^{m}=(P, 0, \ldots, P, 0)$. The $\kappa$ transformation takes the form $\delta \Theta^{\alpha}=-i P^{+} \Gamma^{-\alpha \beta} \kappa_{\beta}$, and thus it follows immediately that $\left(\Gamma^{+} \Theta\right)_{\alpha}=0$.
} 


$$
S=\int d \tau\left[P^{m} \dot{X}_{m}-\frac{i}{2} S^{A} S_{A}+e P^{m} P_{m}\right]
$$

where $S_{A}$ is an $S O(9)$ Majorana spinor, which can be written in terms of $S O(9)$ component of $\Theta^{\alpha}$. The conjugate momentum to $S^{A}$ is

$$
p_{A}=\frac{\partial L}{\partial \dot{S}^{A}}=-\frac{i}{2} S_{A} .
$$

So, the constraints for this gauge-fixed system are

$$
\tilde{D}_{A}=p_{A}+\frac{i}{2} S_{A}
$$

Considering that $\left\{S_{A}, p_{B}\right\}=-i \delta_{A B}$, we obtain

$$
\left\{\tilde{D}_{A}, \tilde{D}_{B}\right\}=\delta_{A B} \text {. }
$$

Hence, the constraint matrix is $C_{A B}=\delta_{A B}$, and its corresponding inverse is $\left(C^{-1}\right)^{A B}=\delta^{A B}$. This allows us to compute the following Dirac bracket:

$$
\begin{aligned}
\left\{S_{A}, S_{B}\right\}_{D} & =\left\{S_{A}, S_{B}\right\}_{P}-\sum_{E, F}\left\{S_{A}, \tilde{D}_{E}\right\}_{P}\left(C^{-1}\right)^{E F}\left\{\tilde{D}_{F}, S_{B}\right\}_{P} \\
& =0-\sum_{E, F}\left(-i \delta_{A E}\right)\left(\delta^{E F}\right)\left(-i \delta_{F B}\right) \\
& =\delta_{A B}
\end{aligned}
$$

As is well known, the representation of the algebra (11) defines the space of physical states. These states will be denoted $|I J\rangle,|B I\rangle$, and $|L M N\rangle$, where we represent $S O(9)$ vector indices by $I, J, K, L, \ldots$, and spinor indices by $A, B, C, D, \ldots$ These states correspond to an $S O(9)$ traceless symmetric tensor, an $S O(9) \Gamma$-traceless vector-spinor, and an $S O(9)$ 3-form, which, together, form the field content of $D=11$ Supergravity. The action of the operators $S_{A}$ on the physical states is defined by

$S_{A}|I J\rangle=\Gamma_{A B}^{I}|B J\rangle+\Gamma^{J}|B I\rangle$

$S_{A}|B I\rangle=\frac{1}{4} \Gamma_{A B}^{J}|I J\rangle+\frac{1}{72}\left(\Gamma_{A B}^{I L M N}+6 \delta^{I L} \Gamma_{A B}^{M N}\right)|L M N\rangle$

$S_{A}|L M N\rangle=\Gamma_{A B}^{L M}|B N\rangle+\Gamma_{A B}^{M N}|B L\rangle+\Gamma_{A B}^{N L}|B M\rangle$.

We can check that these definitions indeed reproduce the desired algebra. Let us check the statement explicitly for the graviton $|I J\rangle$ :

$$
\begin{aligned}
S_{A} S_{B}|I J\rangle= & \Gamma_{B C}^{I} S_{A}|C J\rangle+\Gamma_{B C}^{J} S_{A}|C I\rangle \\
= & \Gamma_{B C}^{I}\left[\frac{1}{4} \Gamma_{A C}^{K}|J K\rangle+\frac{1}{72}\left(\Gamma_{A C}^{J L M N}\right.\right. \\
& \left.\left.+6 \delta^{J L} \Gamma_{A C}^{M N}\right)|L M N\rangle\right]+\Gamma_{B C}^{J}\left[\frac{1}{4} \Gamma_{A C}^{K}|I K\rangle\right. \\
& \left.+\frac{1}{72}\left(\Gamma_{A C}^{I L M N}+6 \delta^{J L} \Gamma_{A C}^{M N}\right)|L M N\rangle\right] .
\end{aligned}
$$

Analogously,

$$
\begin{aligned}
S_{B} S_{A}|I J\rangle= & \Gamma_{A C}^{I} S_{B}|C J\rangle+\Gamma_{A C}^{J} S_{B}|C I\rangle \\
= & \Gamma_{A C}^{I}\left[\frac{1}{4} \Gamma_{B C}^{K}|J K\rangle+\frac{1}{72}\left(\Gamma_{B C}^{J L M N}\right.\right. \\
& \left.\left.+6 \delta^{J L} \Gamma_{B C}^{M N}\right)|L M N\rangle\right]+\Gamma_{A C}^{J}\left[\frac{1}{4} \Gamma_{B C}^{K}|I K\rangle\right. \\
& \left.+\frac{1}{72}\left(\Gamma_{B C}^{I L M N}+6 \delta^{J L} \Gamma_{B C}^{M N}\right)|L M N\rangle\right] .
\end{aligned}
$$

Thus, the anticommutator is

$$
\begin{aligned}
\left\{S_{A}, S_{B}\right\}|I J\rangle= & \frac{1}{4}\left[\Gamma_{B C}^{I} \Gamma_{A C}^{K}+\Gamma_{A C}^{I} \Gamma_{B C}^{K}\right]|J K\rangle+\frac{1}{4}\left[\Gamma_{B C}^{J} \Gamma_{A C}^{K}+\Gamma_{A C}^{J} \Gamma_{B C}^{K}\right]|I K\rangle+\frac{1}{72}\left[\left(\Gamma_{B C}^{I} \Gamma_{A C}^{J L M N}+\Gamma_{B C}^{J} \Gamma_{A C}^{I L M N}+\Gamma_{A C}^{I} \Gamma_{B C}^{J L M N}\right.\right. \\
& \left.\left.+\Gamma_{A C}^{J} \Gamma_{B C}^{I L M N}\right)+6\left(\delta^{J L} \Gamma_{B C}^{I} \Gamma_{A C}^{M N}+\delta^{I L} \Gamma_{B C}^{J} \Gamma_{A C}^{M N}+\delta^{J L} \Gamma_{A C}^{I} \Gamma_{B C}^{M N}+\delta^{I L} \Gamma_{A C}^{J} \Gamma_{B C}^{M N}\right)\right]|L M N\rangle \\
= & \frac{1}{4}\left(2 \delta^{I K} \delta_{A B}|J K\rangle+2 \delta^{J K} \delta_{A B}|I K\rangle\right)+\frac{1}{72}\left[4 !\left(\delta^{I J} \Gamma_{B A}^{L M N]}+\delta^{J[I} \Gamma_{B A}^{L M N]}+\delta^{I J} \Gamma_{A B}^{L M N]}+\delta^{J[I} \Gamma_{A B}^{L M N]}\right)\right. \\
& \left.+6\left(\delta^{J L} \Gamma_{B C}^{I} \Gamma_{A C}^{M N}+\delta^{I L} \Gamma_{B C}^{J} \Gamma_{A C}^{M N}+\delta^{J L} \Gamma_{A C}^{I} \Gamma_{B C}^{M N}+\delta^{I L} \Gamma_{A C}^{J} \Gamma_{B C}^{M N}\right)\right]|L M N\rangle .
\end{aligned}
$$

Now, let us consider the symmetry properties of the $S O(9) \Gamma$-matrices. The 1-form and 4-form are symmetric in their spinor indices, and the 2-form and 3-form are antisymmetric in their spinor indices. Therefore,

$$
\begin{aligned}
\left\{S_{A}, S_{B}\right\}|I J\rangle= & \delta_{A B}|I J\rangle+\frac{1}{12}\left(\delta^{J L} \Gamma_{B C}^{I} \Gamma_{A C}^{M N}+\delta^{I L} \Gamma_{B C}^{J} \Gamma_{A C}^{M N}+\delta^{J L} \Gamma_{A C}^{I} \Gamma_{B C}^{M N}+\delta^{I L} \Gamma_{A C}^{J} \Gamma_{B C}^{M N}\right)|L M N\rangle \\
= & \delta_{A B}|I J\rangle+\frac{1}{12}\left[\delta^{J L}\left(\Gamma_{B A}^{I M N}+\delta^{I[M} \Gamma^{N]}+\Gamma_{A B}^{I M N}+\delta^{I[M} \Gamma_{A B}^{N]}\right)+\delta^{I L}\left(\Gamma_{B A}^{J M N}+\delta^{J[M} \Gamma^{N]}+\Gamma_{A B}^{J M N}+\delta^{J[M} \Gamma_{A B}^{N}\right)\right]|L M N\rangle \\
= & \delta_{A B}|I J\rangle+\frac{1}{12}\left[\delta^{J L} \delta^{I M} \Gamma^{N}-\delta^{J L} \delta^{I N} \Gamma^{M}+\delta^{J L} \delta^{I M} \Gamma^{N}-\delta^{I N} \delta^{J L} \Gamma^{M}\right. \\
& \left.+\delta^{I L} \delta^{J M} \Gamma^{N}-\delta^{I L} \delta^{J N} \Gamma^{M}+\delta^{I L} \delta^{J M} \Gamma^{N}-\delta^{I L} \delta^{J N} \Gamma^{M}\right]|L M N\rangle \\
= & \delta_{A B}|I J\rangle+\frac{1}{12}\left[2 \Gamma^{N}|J I N\rangle-2 \Gamma^{M}|J M I\rangle+2 \Gamma^{N}|I J N\rangle-2 \Gamma^{M}|I M J\rangle\right] \\
= & \delta_{A B}|I J\rangle
\end{aligned}
$$


as expected. One can similarly show that this algebra is satisfied for the action of $S_{A}$ on the other two fields. Therefore, the $D=11$ superparticle spectrum describes the physical degrees of freedom of $D=11$ supergravity.

\section{III. $D=11$ PURE SPINOR SUPERPARTICLE}

As for the $D=10$ case [4], we will obtain the $D=11$ pure spinor superparticle from the gauge-fixed BrinkSchwarz-like superparticle (7) by introducing a new set of variables $\left(\Theta^{\alpha}, P_{\alpha}\right)$ and a new symmetry coming from the following first-class constraints,

$$
\hat{D}_{\alpha}=D_{\alpha}+\frac{1}{\sqrt{\sqrt{2} P^{+}}}\left(\Gamma^{m} \Gamma^{+} S\right)_{\alpha} P_{m},
$$

where $\left\{S_{A}, S_{B}\right\}=\delta_{A B}$ and $D_{\alpha}=P_{\alpha}+\Gamma_{\alpha \beta}^{m} \Theta^{\beta} P_{m}$. Using the relation $\left\{\Theta^{\alpha}, P_{\beta}\right\}=i \delta_{\beta}^{\alpha}$, one can show that $\left\{D_{\alpha}, D_{\beta}\right\}=2 i\left(\Gamma^{m}\right)_{\alpha \beta} P_{m}$. Let us check that these ones are indeed first-class constraints:

$$
\begin{aligned}
\left\{\hat{D}_{\alpha}, \hat{D}_{\beta}\right\} & =\left\{D_{\alpha}, D_{\beta}\right\}+\frac{1}{\sqrt{2} P^{+}}\left(\Gamma^{m} \Gamma^{+}\right)_{\alpha}{ }^{A}\left(\Gamma^{n} \Gamma^{+}\right)_{\beta}{ }^{A} P_{m} P_{n} \\
& =2 i\left(\Gamma^{m}\right)_{\alpha \beta} P_{m}-\frac{\sqrt{2} i}{\sqrt{2} P^{+}} \Gamma_{\alpha \lambda}^{m} \Gamma_{\beta \delta}^{n} \Gamma^{+\lambda \delta} P_{m} P_{n} \\
& =2 i\left(\Gamma^{m}\right)_{\alpha \beta} P_{m}-\frac{i}{P^{+}}\left(\Gamma^{m} \Gamma^{+} \Gamma^{n}\right)_{\alpha \beta} P_{m} P_{n} .
\end{aligned}
$$

Since $\Gamma^{m} \Gamma^{+}=-\Gamma^{+} \Gamma^{m}+2 \eta^{m+}$, we obtain

$$
\begin{aligned}
\left\{\hat{D}_{\alpha}, \hat{D}_{\beta}\right\}= & 2 i\left(\Gamma^{m}\right)_{\alpha \beta} P_{m}-\frac{i}{P^{+}}\left(2 \eta^{+m} \Gamma_{\alpha \beta}^{n}\right) P_{m} P_{n} \\
& +\frac{i}{P^{+}} \Gamma_{\alpha \beta}^{+} P^{2} \\
= & \frac{i}{P^{+}} \Gamma_{\alpha \beta}^{+} P^{2} .
\end{aligned}
$$

Thus, the modified Brink-Schwarz-like action will be

$$
S=\int d \tau\left(\dot{X}^{m} P_{m}-\frac{i}{2} \dot{S}^{A} S_{A}+e P^{m} P_{m}+\dot{\Theta}^{\alpha} P_{\alpha}+f^{\alpha} \hat{D}_{\alpha}\right),
$$

where we have added the usual kinetic term for the variables $\left(\Theta^{\alpha}, P_{\alpha}\right)$ and the last term takes into account the new constraint through the fermionic Lagrange multiplier $f^{\alpha}$. The standard BRST method gives us the following gauge-fixed action,

$$
S=\int d \tau\left(\dot{X}^{m} P_{m}-\frac{i}{2} \dot{S}^{A} S_{A}-\frac{1}{2} P^{m} P_{m}+\dot{\Theta}^{\alpha} P_{\alpha}+\dot{c} b+\dot{\hat{\Lambda}}^{\alpha} \hat{W}_{\alpha}\right),
$$

and the BRST operator

$$
\hat{Q}=\hat{\Lambda}^{\alpha} \hat{D}_{\alpha}+c P^{m} P_{m}-\frac{i}{2 P^{+}}\left(\hat{\Lambda} \Gamma^{+} \hat{\Lambda}\right) b
$$

once we choose the gauge $e=-\frac{1}{2}$ and $f^{\alpha}=0$. The ghosts $c$ and $\hat{\Lambda}^{\alpha}$ come from gauge fixing the reparametrization symmetry and the new fermionic symmetry, respectively.

Now, we will show that the cohomology of the BRST operator $\hat{Q}$ is equivalent to the cohomology of a BRST operator $Q=\Lambda^{\alpha} D_{\alpha}$, where $\Lambda^{\alpha}$ is a pure spinor. We will show this claim in two steps. First, we show that the $\hat{Q}$-cohomology is equivalent to $Q^{\prime}$-cohomology, where $Q^{\prime}=\Lambda^{\alpha} \hat{D}_{\alpha}$ and $\Lambda^{\prime} \Gamma^{+} \Lambda^{\prime}=0$. Finally, we will prove that the $Q^{\prime}$-cohomology is equivalent to the $Q$-cohomology.

Let us start by defining the operator $Q_{0}=\Lambda_{0}^{\alpha} \hat{D}_{\alpha}$. Notice that when $\Lambda_{0}^{\alpha}$ is equal to $\hat{\Lambda}^{\alpha}$ or $\Lambda^{\prime \alpha}, Q_{0}$ becomes the first term of $\hat{Q}$ or $Q^{\prime}$, respectively. Now, let $V$ be a state such that $Q_{0} V=\left(\Lambda_{0} \Gamma^{+} \Lambda_{0}\right) W$, for some W. Because of the property that $\Lambda^{\prime \alpha}$ satisfies, $\mathrm{V}$ is annihilated by $Q^{\prime}$. Also, using (19), we find that $\left(Q_{0}\right)^{2}=\frac{i}{2 P^{+}} P^{m} P_{m}\left(\Lambda_{0} \Gamma^{+} \Lambda_{0}\right)$. So, we conclude that $Q_{0} W=\frac{i}{2 P^{+}} P^{m} P_{m} V$. We can then show that the state $\hat{V}=V-2 i P^{+} c W$ is annihilated by $\hat{Q}$,

$$
\begin{aligned}
\hat{Q} \hat{V}= & \hat{Q}\left(V-2 i P^{+} c W\right) \\
= & \hat{Q} V-2 i P^{+}(\hat{Q} c) W+2 i P^{+} c(\hat{Q} W) \\
= & \left(\hat{\Lambda} \Gamma^{+} \hat{\Lambda}\right) W+c P^{m} P_{m} V-2 i P^{+}\left(-\frac{i}{2 P^{+}}\right)\left(\hat{\Lambda} \Gamma^{+} \hat{\Lambda}\right) W \\
& +2 i P^{+} c\left(\frac{i}{2 P^{+}}\right) P^{m} P_{m} V \\
= & \left(\hat{\Lambda} \Gamma^{+} \hat{\Lambda}\right) W+c P^{m} P_{m} V-\left(\hat{\Lambda} \Gamma^{+} \hat{\Lambda}\right) W-c P^{m} P_{m} V \\
= & 0,
\end{aligned}
$$

where we have assumed that $b$ annihilates physical states. Now, let us show that if a state $V$ is BRST trivial (in the $Q^{\prime}$ cohomology) we can find a state $\hat{V}=V-2 i P^{+} c W$ which is also BRST trivial (in the $\hat{Q}$-cohomology). Let $V$ be a state which satisfies $V=Q_{0} \Omega+\left(\Lambda_{0} \Gamma^{+} \Lambda_{0}\right) Y$, for some $Y$. It is clear that if $\Lambda_{0}^{\alpha}=\Lambda^{\prime \alpha}$ we have that $V$ is $Q^{\prime}$ exact and if $\Lambda_{0}^{\alpha}=\hat{\Lambda}^{\alpha}$ we have that the first term of $\hat{Q}$ is equal to $V-\left(\hat{\Lambda} \Gamma^{+} \hat{\Lambda}\right) Y$. So, we see that

$$
\begin{aligned}
\hat{Q}\left(\Omega+2 i P^{+} c Y\right)= & \hat{Q} \Omega+2 i P^{+}(\hat{Q} c) Y-2 i P^{+} c(\hat{Q} Y) \\
= & V-\left(\hat{\Lambda} \Gamma^{+} \hat{\Lambda}\right) Y+c P^{m} P_{m} \\
& +2 i P^{+}\left(-\frac{i}{2 P^{+}}\right)\left(\hat{\Lambda} \Gamma^{+} \hat{\Lambda}\right) Y \\
& -2 i P^{+} c\left(W-\frac{i}{2 P^{+}} P^{m} P_{m} \Omega\right),
\end{aligned}
$$


where we used the fact that $b$ annihilates $\Omega$ as well as the result $\hat{Q}_{0} Y=W-\frac{i}{2 P^{+}} P^{m} P_{m}$, which follows from the definition of $V$. Hence, we obtain

$$
\begin{aligned}
\hat{Q}\left(\Omega+2 i P^{+} c Y\right)= & V-\left(\hat{\Lambda} \Gamma^{+} \hat{\Lambda}\right) Y+c P^{m} P_{m} \Omega \\
& +\left(\hat{\Lambda} \Gamma^{+} \hat{\Lambda}\right) Y-2 i P^{+} c W-c P^{m} P_{m} \Omega \\
= & V-2 i P^{+} c W \\
= & \hat{V} .
\end{aligned}
$$

Therefore, we have proven that for each state $V$ in the $Q^{\prime}$-cohomology we can find a state $\hat{V}$ in the $\hat{Q}$-cohomology. If we reverse the arguments given above, we can show that any state in the $\hat{Q}$-cohomology corresponds to a state in the $Q^{\prime}$-cohomology.

The last step is to show that the $Q^{\prime}$-cohomology is equivalent to the $Q$-cohomology. We will do this by using two different approaches.

\section{A. Group decomposition $S O(9) \rightarrow S U(2) \times S U(4)$}

The $S O(10,1)$ spinors $\Lambda^{\alpha}$ and $D_{\alpha}$ can be expressed in terms of their $S O(8)$ components in the following way,

$$
\Lambda^{\prime \alpha}=\left(\begin{array}{c}
\lambda^{\prime a} \\
\lambda^{\prime a} \\
\tilde{\lambda}^{\prime a} \\
\tilde{\lambda}^{\prime a}
\end{array}\right), \quad D_{\alpha}=\left(\begin{array}{c}
\tilde{d}^{a} \\
\tilde{d}^{a} \\
-d^{a} \\
-d^{a}
\end{array}\right),
$$

where $a, \dot{a}=1, \ldots, 8$. The constraint $\Lambda^{\prime} \Gamma^{+} \Lambda^{\prime}=0$ can be written in terms of these $S O(8)$ components as follows:

$$
\lambda^{\prime a} \lambda^{\prime a}+\tilde{\lambda}^{\prime a} \tilde{\lambda}^{\prime a}=0
$$

The particular representation for $S O(10,1) \Gamma$-matrices used in this section is studied in detail in Appendix A. Now, we find it useful to break $S O(9)$ into $S U(2) \times S U(4)$. The branching rule for the spinor representation is $16 \rightarrow(2,4)+(2, \overline{4})$. Explicit expressions for the $S U(2) \times$ $S U(4)$ components corresponding to $S^{a}, \bar{S}^{\dot{a}}, d^{\dot{a}}, \tilde{d}^{a}, \lambda^{\prime a}$, and $\tilde{\lambda}^{\prime a}$ are given below, Eq. (28). There, the $S O(9)$ spinor $S_{A}$ has been expressed in terms of its $S O(8)$ components $S^{a}$, $\bar{S}^{\dot{a}}$; and $\hat{A}, \overline{\hat{A}}=1, \ldots, 4$. It should be clear in (28) that fields in the same representation of $S U(4)$ (4 or $\overline{4})$ form $S U(2)$ doublets. So, for instance, $\left(\begin{array}{c}d_{\hat{A}} \\ \tilde{d}_{\hat{A}}\end{array}\right)$ transforms under $(2,4),\left(\begin{array}{c}\lambda_{\overline{\hat{A}}}^{\prime} \\ \tilde{\lambda}_{\overline{\hat{A}}}^{\prime}\end{array}\right)$ transforms under $(2, \overline{4})$, etc. Notice that the representations 4 and $\overline{4}$ are defined by the null spinor $\left(\Gamma^{+} \Lambda^{\prime}\right)^{A}$ by using the fact that one can always choose an $S U$ (4) subgroup under which this spinor is invariant. Therefore, we define the antifundamental representation (4) in such a way that $\left(\Gamma^{J}\right)_{(\Upsilon \overline{\hat{A}}) A}\left(\Gamma^{+} \Lambda^{\prime}\right)^{A}=0$, where $J=1, \ldots, 9, \Upsilon$ is an $S U(2)$ vector index and $A$ is an $S O(9)$ spinor index,

$$
\begin{array}{lll}
S_{\hat{A}}=\frac{1}{\sqrt{2}}\left(S^{2 a}+i S^{2 a-1}\right) & d_{\hat{A}}=\frac{1}{\sqrt{2}}\left(d^{2 \dot{a}}+i d^{2 \dot{a}-1}\right) & \lambda_{\hat{A}}^{\prime}=\frac{1}{\sqrt{2}}\left(\lambda^{\prime 2 a}+i \lambda^{\prime 2 a-1}\right) \\
S_{\overline{\hat{A}}}=\frac{1}{\sqrt{2}}\left(S^{2 a}-i S^{2 a-1}\right) & d_{\overline{\hat{A}}}=\frac{1}{\sqrt{2}}\left(d^{2 \dot{a}}-i d^{2 \dot{a}-1}\right) & \lambda_{\hat{\hat{A}}}^{\prime}=\frac{1}{\sqrt{2}}\left(\lambda^{\prime 2 a}-i \lambda^{\prime 2 a-1}\right) \\
\tilde{S}_{\hat{A}}=\frac{1}{\sqrt{2}}\left(\bar{S}^{2 \dot{a}}+i \bar{S}^{2 \dot{a}-1}\right) & \tilde{d}_{\hat{A}}=\frac{1}{\sqrt{2}}\left(\tilde{d}^{2 a}+i \tilde{d}^{2 a-1}\right) & \tilde{\lambda}_{\hat{A}}^{\prime}=\frac{1}{\sqrt{2}}\left(\tilde{\lambda}^{\prime 2 \dot{a}}+i \tilde{\lambda}^{\prime 2 \dot{a}-1}\right) \\
\tilde{S}_{\overline{\hat{A}}}=\frac{1}{\sqrt{2}}\left(\bar{S}^{2 \dot{a}}-i \bar{S}^{2 \dot{a}-1}\right) & \tilde{d}_{\overline{\hat{A}}}=\frac{1}{\sqrt{2}}\left(\tilde{d}^{2 a}-i \tilde{d}^{2 a-1}\right) & \tilde{\lambda}_{\overline{\hat{A}}}^{\prime}=\frac{1}{\sqrt{2}}\left(\tilde{\lambda}^{\prime 2 \dot{a}}-i \tilde{\lambda}^{\prime 2 \dot{a}-1}\right) .
\end{array}
$$

After performing the following shifts,

$$
\begin{gathered}
S_{\hat{A}} \rightarrow S_{\hat{A}}-\left(\frac{\sqrt{\sqrt{2}}}{2 \sqrt{P^{+}}}\right) \tilde{d}_{\hat{A}} \\
\tilde{S}_{\hat{A}} \rightarrow \tilde{S}_{\hat{A}}+\left(\frac{\sqrt{\sqrt{2}}}{2 \sqrt{P^{+}}}\right) d_{\hat{A}},
\end{gathered}
$$

the operator $Q^{\prime}$ will change by the similarity transformation

$$
Q^{\prime} \rightarrow e^{-\left[K\left(S_{\tilde{A}} \tilde{d}_{\hat{A}}-\tilde{S}_{\bar{A}} d_{\hat{A}}\right)\right]} Q^{\prime} e^{\left[K\left(S_{\tilde{A}} \tilde{d}_{\hat{A}}-\tilde{S}_{\bar{A}} d_{\hat{A}}\right)\right]},
$$

where $K=-\frac{\sqrt{\sqrt{2}}}{2 \sqrt{P+}}$. This result can be expanded by using the Baker-Campbell-Hausdorff $(\mathrm{BCH})$ formula

$$
e^{-Z} X e^{Z}=X+[X, Z]+\frac{1}{2}[[X, Z], Z]+\ldots,
$$

where $X=Q^{\prime}=\Lambda^{\prime \alpha} \hat{D}_{\alpha}$ and $Z=K\left(S_{\overline{\hat{A}}} \tilde{d}_{\hat{A}}-\tilde{S}_{\overline{\hat{A}}} d_{\hat{A}}\right)$. The first term is just $Q^{\prime}$, which can be cast as 


$$
\begin{aligned}
& Q^{\prime}=\Lambda^{\prime \alpha} D_{\alpha}+\frac{1}{\sqrt{\sqrt{2} P^{+}}}\left(\Lambda^{\prime} \Gamma^{m} \Gamma^{+} S\right) P_{m} \\
& =\lambda_{\dot{a}}^{\prime} \tilde{d}_{\dot{a}}+\lambda_{\overline{\hat{A}}}^{\prime} \tilde{d}_{\hat{A}}+\lambda_{\hat{A}}^{\prime} \tilde{d}_{\overline{\hat{A}}}-\tilde{\lambda}_{a}^{\prime} d_{a}-\tilde{\lambda}_{\overline{\hat{A}}}^{\prime} d_{\hat{A}}-\tilde{\lambda}_{\hat{A}}^{\prime} d_{\overline{\hat{A}}}+\sqrt{2 \sqrt{2} P^{+}} \lambda_{\overline{\hat{A}}}^{\prime} S_{\hat{A}}+\sqrt{2 \sqrt{2} P^{+}} \lambda_{\hat{A}}^{\prime} S_{\overline{\hat{A}}} \\
& +\sqrt{2 \sqrt{2} P^{+}} \tilde{\lambda}_{\overline{\hat{A}}}^{\prime} \tilde{S}_{\hat{A}}+\sqrt{2 \sqrt{2} P^{+}} \tilde{\lambda}_{\hat{A}^{\prime}} \tilde{S}_{\overline{\hat{A}}}+\sqrt{\frac{\sqrt{2}}{P^{+}}}\left[\lambda^{\prime \dot{a}}\left(\sigma^{\hat{i}}\right)_{\dot{a} \hat{A}} S_{\overline{\hat{A}}} P_{\hat{i}}-\tilde{\lambda}^{\prime a}\left(\sigma^{\hat{i}}\right)_{\hat{A} a} \tilde{S}_{\overline{\hat{A}}} P_{\hat{i}}\right] \\
& \left.+\sqrt{\frac{\sqrt{2}}{P^{+}}} \tilde{\lambda}_{\hat{A}}^{\prime} S_{\overline{\hat{A}}}+\lambda_{\hat{A}}^{\prime} \tilde{S}_{\overline{\hat{A}}}\right] P^{11} .
\end{aligned}
$$

To find the second term in (32), it is necessary to compute the $S U(4)$ (anti)commutation relations, which can be obtained from the $S O(8)$ relations:

$$
\begin{array}{cc}
\left\{\tilde{d}^{a}, \tilde{d}^{b}\right\}=-2 \sqrt{2} \delta^{a b} P^{+}, & \left\{d^{a}, \tilde{d}^{b}\right\}=2 \delta^{a b} P^{11} \\
\left\{d^{\dot{a}}, d^{\dot{b}}\right\}=-2 \sqrt{2} \delta^{\dot{a} \dot{b}} P^{+}, & \left\{\tilde{d}^{\dot{a}}, d^{\dot{b}}\right\}=2 \delta^{\dot{a} \dot{b}} P^{11} \\
\left\{d^{a}, d^{b}\right\}=-2 \sqrt{2} \delta^{a b} P^{-}, \quad\left\{d^{a}, d^{\dot{b}}\right\}=2\left(\sigma^{\hat{i}}\right)^{a \dot{b}} P^{\hat{i}} \\
\left\{\tilde{d}^{\dot{a}}, \tilde{d}^{\dot{b}}\right\}=-2 \sqrt{2} \delta^{\dot{a} \dot{b}} P^{-}, \quad\left\{\tilde{d}^{\dot{a}}, d^{b}\right\}=-2\left(\sigma^{\hat{i}}\right)^{\dot{a} b} P^{\hat{i}} .
\end{array}
$$

Using these, together with (28), leads us to the $S U(4)$ relations displayed below, Eq. (35):

$$
\begin{aligned}
& \left\{S_{\hat{A}}, S_{\overline{\hat{A}}}\right\}=\eta_{\hat{A} \overline{\hat{A}}}, \quad\left\{\tilde{d}_{\dot{a}}, d_{\hat{A}}\right\}=2 \delta_{\dot{a} A} P^{11}, \quad\left\{d_{a}, d_{\hat{A}}\right\}=2\left(\sigma^{\hat{i}}\right)_{a \hat{A}} P^{\hat{i}} \\
& \left\{\tilde{S}_{\hat{A}}, \tilde{S}_{\overline{\hat{A}}}\right\}=\eta_{\hat{A} \bar{A}}, \quad\left\{\tilde{d}_{\dot{a}}, d_{\overline{\hat{A}}}\right\}=2 \delta_{\dot{a} \bar{A}} P^{11}, \quad\left\{d_{a}, d_{\overline{\hat{A}}}\right\}=2\left(\sigma^{\hat{i}}\right)_{a \overline{\hat{A}}} P^{\hat{i}} \\
& \left\{d_{\hat{A}}, d_{\overline{\hat{A}}}\right\}=-2 \sqrt{2} \eta_{\hat{A} \overline{\hat{A}}} P^{+}, \quad\left\{d_{a}, \tilde{d}_{\overline{\hat{A}}}\right\}=2 \delta_{a \bar{A}} P^{11}, \quad\left\{\tilde{d}_{\dot{a}}, \tilde{d}_{\overline{\hat{A}}}\right\}=-2\left(\sigma^{\hat{i}}\right)_{\dot{a} \overline{\hat{A}}} P^{\hat{i}} . \\
& \left\{\tilde{d}_{\hat{A}}, \tilde{d}_{\overline{\hat{A}}}\right\}=-2 \sqrt{2} \eta_{\hat{A} \overline{\hat{A}}} P^{+}, \quad\left\{d_{a}, \tilde{d}_{\hat{A}}\right\}=2 \delta_{a A} P^{11}, \quad\left\{\tilde{d}_{\dot{a}}, \tilde{d}_{\hat{A}}\right\}=-2\left(\sigma^{\hat{i}}\right)_{\dot{a} \hat{A}} P^{\hat{i}}
\end{aligned}
$$

Hence, we get

$$
\begin{aligned}
K\left[Q^{\prime}, S_{\overline{\hat{A}}} \tilde{d}_{\hat{A}}-\tilde{S}_{\overline{\hat{A}}} d_{\hat{A}}\right]= & -\sqrt{2 \sqrt{2} P^{+}} \lambda_{\hat{A}}^{\prime} S_{\overline{\hat{A}}}-\sqrt{2 \sqrt{2} P^{+}} \tilde{\lambda}_{\hat{A}}^{\prime} \tilde{S}_{\overline{\hat{A}}}-\lambda_{\hat{\hat{A}}}^{\prime} \tilde{d}_{\hat{A}}+\tilde{\lambda}_{\hat{\hat{A}}}^{\prime} d_{\hat{A}}-\sqrt{\frac{\sqrt{2}}{P^{+}}} \tilde{\lambda}_{\hat{A}}^{\prime} S_{\overline{\hat{A}}} P^{11} \\
& -\sqrt{\frac{\sqrt{2}}{P^{+}}} \lambda_{\hat{A}}^{\prime} \tilde{S}_{\overline{\hat{A}}} P^{11}-\sqrt{\frac{\sqrt{2}}{P^{+}}} \lambda^{\prime}\left(\sigma^{\hat{i}}\right)_{\dot{a} \hat{A}} S_{\overline{\hat{A}}} P_{\hat{i}}+\sqrt{\frac{\sqrt{2}}{P^{+}}} \tilde{\lambda}^{\prime a}\left(\sigma^{\hat{i}}\right)_{\hat{A} a} \tilde{S}_{\bar{A}} P_{\hat{i}} .
\end{aligned}
$$

From this expression, it is easy to see that

$$
\left[\left[Q^{\prime}, Z\right], Z\right]=0,
$$

and so the third term and all of the other ones in (32) (which were represented by ...) vanish.

Therefore, we have arrived at the following result,

$$
Q^{\prime} \rightarrow \lambda_{\dot{a}}^{\prime} \tilde{d}_{\dot{a}}+\lambda_{\hat{A}}^{\prime} \tilde{d}_{\overline{\hat{A}}}-\tilde{\lambda}_{a}^{\prime} d_{a}-\tilde{\lambda}_{\hat{A}}^{\prime} d_{\overline{\hat{A}}}+\sqrt{2 \sqrt{2} P^{+}} \lambda_{\overline{\hat{A}}}^{\prime} S_{\hat{A}}+\sqrt{2 \sqrt{2} P^{+}} \tilde{\lambda}_{\overline{\hat{A}}}^{\prime} \tilde{S}_{\hat{A}},
$$

where $\lambda^{\prime a}$ and $\tilde{\lambda}^{\prime a}$ satisfy the relation $\lambda^{\dot{a}} \lambda^{\dot{a}}+\tilde{\lambda}^{\prime a} \tilde{\lambda}^{\prime a}=0$. If we define a spinor, $\Lambda^{\alpha}=\left[\lambda_{\hat{A}}, \lambda_{\overline{\hat{A}}}, \lambda_{\dot{a}}, \tilde{\lambda}_{a}, \tilde{\lambda}_{\hat{A}}, \tilde{\lambda}_{\overline{\hat{A}}}\right]=$ $\left[\lambda_{\hat{A}}^{\prime}, 0, \lambda_{\dot{a}}^{\prime}, \tilde{\lambda}_{a}^{\prime}, \tilde{\lambda}_{\hat{A}}^{\prime}, 0\right]$, the previous expression can be written as

$$
Q^{\prime} \rightarrow \Lambda^{\alpha} D_{\alpha}+\sqrt{2 \sqrt{2} P^{+}} \lambda_{\overline{\hat{A}}}^{\prime} S_{\hat{A}}+\sqrt{2 \sqrt{2} P^{+}} \tilde{\lambda}_{\overline{\hat{A}}}^{\prime} \tilde{S}_{\hat{A}}
$$

Furthermore, after using the quartet argument [11], it is clear that the $Q^{\prime}$-cohomology is equivalent to the $Q$-cohomology,

$$
Q^{\prime} \rightarrow Q=\Lambda^{\alpha} D_{\alpha},
$$

where $\Lambda^{\alpha}$ is a pure spinor.

\footnotetext{
${ }^{4}$ That is, the states in the Hilbert space will be independent of $\lambda_{\overline{\hat{A}}}^{\prime}, S_{\hat{A}}, \tilde{\lambda}_{\overline{\hat{A}}}^{\prime}$, and $\tilde{S}_{\hat{A}}$ and their respective conjugate momenta $w_{\hat{A}}^{\prime}, S_{\overline{\hat{A}}}, \tilde{w}_{\hat{A}}^{\prime}$, and $\tilde{S}_{\overline{\hat{A}}}$.
} 


\section{B. Group decomposition $\mathrm{SO}(9) \rightarrow U(1) \times S O(7)$}

We will express $S O(10,1)$ spinors in terms of their $S O(3,1) \times S O(7)$ components,

$$
\chi^{\alpha}=\left(\begin{array}{c}
\chi^{ \pm \pm 0} \\
\chi^{ \pm \pm i}
\end{array}\right)
$$

where $i=1, \ldots, 7$. The notation \pm and the representation of the $S O(10,1)$ gamma matrices used here are explained in detail in Appendix B. Using this notation, we can express the (anti)commutation relations studied above in the $S O(3,1) \times S O(7)$ language

$$
\begin{aligned}
\left\{D^{--0}, D^{-+0}\right\} & =2 \sqrt{2} P^{+}, \quad\left\{D^{--0}, D^{+-0}\right\}=2 \sqrt{2} P^{2+3 i}, \quad\left\{D^{--i}, D^{++0}\right\}=-2 P^{i} \\
\left\{D^{--i}, D^{-+j}\right\} & =-2 \sqrt{2} P^{+} \delta^{i j}, \quad\left\{D^{--i}, D^{+-j}\right\}=-2 \sqrt{2} P^{2+3 i} \delta^{i j}, \quad\left\{D^{++i}, D^{--0}\right\}=2 P^{i} \\
\left\{D^{++0}, D^{+-0}\right\} & =2 \sqrt{2} P^{-}, \quad\left\{D^{++0}, D^{-+0}\right\}=2 \sqrt{2} P^{2-3 i}, \quad\left\{D^{-+i}, D^{+-0}\right\}=2 P^{i} \\
\left\{D^{++i}, D^{+-j}\right\} & =-2 \sqrt{2} P^{-} \delta^{i j}, \quad\left\{D^{++i}, D^{-+j}\right\}=-2 \sqrt{2} P^{2-3 i} \delta^{i j}, \quad\left\{D^{+-i}, D^{-+0}\right\}=-2 P^{i}
\end{aligned}
$$

and also

$$
\begin{aligned}
\left\{S^{--0}, S^{-+0}\right\} & =-1 \\
\left\{S^{--i}, S^{-+j}\right\} & =\delta^{i j},
\end{aligned}
$$

and any other anticommutator vanishes. Under a certain subgroup $U(1) \times S O(7) \subset S O(9)$, the null spinor $\left(\Gamma^{+} \Lambda^{\prime}\right)^{A}$ will be invariant up to rescaling. This subgroup is chosen in such a way that $\left(\Gamma^{2+3 i}\right)_{(-0) A}\left(\Gamma^{+} \Lambda^{\prime}\right)^{A}=\left(\Gamma^{j}\right)_{(-0) A}\left(\Gamma^{+} \Lambda^{\prime}\right)^{A}=0$, where we have dropped out the minus sign associated to the first $U(1)$ charge, and $j=1, \ldots, 7$.

The BRST operator $Q^{\prime}$ can be expressed in terms of $S O(3,1) \times S O(7)$ variables:

$$
\begin{aligned}
Q^{\prime}= & \Lambda^{\prime \alpha} D_{\alpha}+\frac{1}{\sqrt{\sqrt{2} P^{+}}}\left[-\left(\Lambda^{\prime} \Gamma^{-} \Gamma^{+} S\right) P^{+}+\left(\Lambda^{\prime} \Gamma^{2-3 i} \Gamma^{+} S\right) P^{2+3 i}+\left(\Lambda^{\prime} \Gamma^{2+3 i} \Gamma^{+} S\right) P^{2-3 i}+\left(\Lambda^{\prime} \Gamma^{j} \Gamma^{+} S\right) P^{j}\right] \\
= & \Lambda^{\prime \alpha} D_{\alpha}-\frac{2 \sqrt{P^{+}}}{\sqrt{\sqrt{2}}}\left(\Lambda^{\prime+-0} S^{-+0}-\Lambda^{\prime+-i} S^{-+i}+\Lambda^{\prime++0} S^{--0}-\Lambda^{\prime++i} S^{--i}\right) \\
& +\sqrt{\frac{2 \sqrt{2}}{P^{+}}}\left(\Lambda^{-+0} S^{-+0}\right) P^{2+3 i}-\sqrt{\frac{2 \sqrt{2}}{P^{+}}}\left(\Lambda^{--j} S^{--j}\right) P^{2-3 i}-\sqrt{\frac{\sqrt{2}}{P^{+}}}\left(\Lambda^{--j} S^{-+0}\right) P^{j} \\
& -\sqrt{\frac{\sqrt{2}}{P^{+}}}\left(\Lambda^{-+0} S^{--j}\right) P^{j}
\end{aligned}
$$

After performing the following shifts,

$$
\begin{aligned}
& S^{--0} \rightarrow S^{--0}-\frac{\sqrt{\sqrt{2}}}{2 \sqrt{P^{+}}} D^{--0} \\
& S^{-+i} \rightarrow S^{-+i}-\frac{\sqrt{\sqrt{2}}}{2 \sqrt{P^{+}}} D^{-+i},
\end{aligned}
$$

the BRST operator will change by

$$
Q^{\prime} \rightarrow e^{-Z} Q^{\prime} e^{Z}
$$

where $Z=\frac{\sqrt{\sqrt{2}}}{2 \sqrt{P^{+}}}\left(S^{-+0} D^{--0}-S^{--i} D^{-+i}\right)$. The BCH formula (32) gives us the result 


$$
\begin{aligned}
Q^{\prime} \rightarrow & Q^{\prime}+\left[Q^{\prime}, Z\right]+\frac{1}{2}\left[\left[Q^{\prime}, Z\right], Z\right]+\cdots \\
\rightarrow & -\Lambda^{\prime++0} D^{--0}+\Lambda^{\prime++i} D^{--i}+\Lambda^{\prime--0} D^{++0}-\Lambda^{\prime-i} D^{++i}-\Lambda^{\prime+-0} D^{-+0}+\Lambda^{\prime+-i} D^{-+i} \\
& +\Lambda^{\prime-+0} D^{+-0}-\Lambda^{\prime-+i} D^{+-i}-\frac{2 \sqrt{P^{+}}}{\sqrt{\sqrt{2}}}\left(\Lambda^{\prime+-0} S^{-+0}-\Lambda^{\prime++i} S^{--i}+\Lambda^{\prime++0} S^{--0}-\Lambda^{\prime+-i} S^{-+i}\right) \\
& +\sqrt{\frac{2 \sqrt{2}}{P^{+}}}\left(\Lambda^{-+0} S^{-+0}\right) P^{2+3 i}-\sqrt{\frac{2 \sqrt{2}}{P^{+}}}\left(\Lambda^{--j} S^{--j}\right) P^{2-3 i} \\
& -\sqrt{\frac{\sqrt{2}}{P^{+}}}\left(\Lambda^{--j} S^{-+0}\right) P^{j}-\sqrt{\frac{\sqrt{2}}{P^{+}}}\left(\Lambda^{-+0} S^{--j}\right) P^{j}+\frac{\sqrt{\sqrt{2}}}{2 \sqrt{P^{+}}}\left[2 \sqrt{2} \Lambda^{\prime+-0} S^{-+0} P^{+}\right. \\
& +2 \Lambda^{\prime--i} S^{-+0} P^{i}-2 \sqrt{2} \Lambda^{\prime-+0} S^{-+0} P^{2+3 i}-2 \sqrt{2} P^{+} \Lambda^{\prime++i} S^{--i}+2 \sqrt{2} P^{2-3 i} \Lambda^{\prime--i} S^{--i} \\
& \left.+2 \Lambda^{\prime-+0} S^{--i} P^{i}\right]+\Lambda^{\prime++0} D^{--0}-\Lambda^{\prime+-i} D^{-+i}+\cdots,
\end{aligned}
$$

where the ellipsis represents $\frac{1}{2 !}\left[\left[Q^{\prime}, Z\right], Z\right]+\frac{1}{3 !}\left[\left[\left[Q^{\prime}, Z\right], Z\right], Z\right]+\cdots$. However, these terms vanish because $\left[\left[Q^{\prime}, Z\right], Z\right]=0$, as can be seen from Eq. (42). Thus, we are left with

$$
Q^{\prime} \rightarrow \Lambda^{\prime++i} D^{--i}+\Lambda^{\prime--0} D^{++0}-\Lambda^{\prime--i} D^{++i}-\Lambda^{\prime+-0} D^{-+0}+\Lambda^{\prime-+0} D^{+-0}-\Lambda^{\prime-+i} D^{+-i}-\sqrt{2 \sqrt{2} P^{+}}\left(\Lambda^{\prime++0} S^{--0}-\Lambda^{\prime+-i} S^{-+i}\right) .
$$

If we define a spinor $\Lambda^{\alpha}=\left[\Lambda^{++0}, \Lambda^{++i}, \Lambda^{--0}, \Lambda^{--i}, \Lambda^{+-0}, \Lambda^{+-i}, \Lambda^{-+0}, \Lambda^{-+i}\right]=\left[0, \Lambda^{\prime++i}, \Lambda^{\prime--0}, \Lambda^{\prime--i}, \Lambda^{\prime+-0}, 0, \Lambda^{\prime-+0}, \Lambda^{\prime-+i}\right]$ where $\Lambda^{\prime} \Gamma^{+} \Lambda^{\prime}=0$, the resulting BRST operator can be written as

$Q^{\prime} \rightarrow \Lambda^{\alpha} D_{\alpha}-\sqrt{2 \sqrt{2} P^{+}}\left(\Lambda^{\prime++0} S^{-+0}-\Lambda^{\prime+-i} S^{--i}\right)$

From this last expression, we can conclude that the space of physical states will not depend on the canonical variables $S^{-+0}, S^{--i}, \Lambda^{\prime++0}$, and $\Lambda^{\prime+-i}$ and their respective conjugate momenta $S^{--0}, S^{-+i}, W^{\prime--0}$, and $W^{\prime-+i}$. Therefore, the BRST operator takes the simple form

$$
Q^{\prime} \rightarrow Q=\Lambda^{\alpha} D_{\alpha}
$$

where $\Lambda^{\alpha}$ is a $D=11$ pure spinor. In this way, we have shown that the modified Brink-Schwarz-like superparticle action (21) is equivalent to the theory described by the manifestly Lorentz covariant action

$$
S=\int d \tau\left(\dot{X}^{m} P_{m}-\frac{1}{2} P^{m} P_{m}+\dot{\Theta}^{\alpha} P_{\alpha}+\dot{\Lambda}^{\alpha} W_{\alpha}\right)
$$

and the BRST operator $Q=\Lambda^{\alpha} D_{\alpha}$, where $\Lambda \Gamma^{m} \Lambda=0$. This theory is the $D=11$ pure spinor superparticle.

\section{LIGHT-CONE ANALYSIS OF THE PURE SPINOR COHOMOLOGY}

In this section, it will be shown that the pure spinor physical condition implies light-cone equations of motion for $D=11$ linearized supergravity in $D=9$ superspace, which coincide with those found in Ref. [7]. To see this, let us write $Q$ in $S O(9)$ notation (see Appendix A),

$$
Q=\Lambda^{A} D_{A}+\bar{\Lambda}^{A} \bar{D}_{A},
$$

and define the operator

$$
R=\frac{P^{I} \bar{N}_{I}}{\sqrt{2} P^{+}}
$$

where $I=1, \ldots, 8,11$ and $\bar{N}^{I}=\Lambda^{A} \Gamma_{A B}^{I} \bar{W}^{B}$. The corresponding similarity transformation generated by this operator is

$$
\begin{aligned}
\tilde{Q} & =e^{-R} Q e^{R} \\
& =Q+[Q, R]+\frac{1}{2}[[Q, R], R]+\cdots \\
& =\Lambda^{A} D_{A}+\bar{\Lambda}^{A} \bar{D}_{A}+\frac{i}{\sqrt{2} P^{+}} P_{I}\left(\Lambda^{A} \Gamma_{A B}^{I} \bar{D}^{B}\right) \\
& =\Lambda^{A}\left[D_{A}+\frac{i}{\sqrt{2} P^{+}} P_{I}\left(\Gamma^{I} \bar{D}\right)_{A}\right]+\bar{\Lambda}^{A} \bar{D}_{A} \\
& =\Lambda^{A} G_{A}+\bar{\Lambda}^{A} \bar{D}_{A},
\end{aligned}
$$

where $G_{A}$ is defined by the relation 


$$
\begin{aligned}
G_{A} & =D_{A}+\frac{i}{\sqrt{2} P^{+}} P_{I}\left(\Gamma^{I} \bar{D}\right)_{A} \\
& =D_{A}+\frac{1}{\sqrt{2} P^{+}} P_{\hat{i}}\left(\gamma^{9} \gamma^{\hat{i}} \bar{D}\right)_{A}-\frac{1}{\sqrt{2} P^{+}} P_{11} \bar{D}_{A},
\end{aligned}
$$

where $\hat{i}$ is an $S O(8)$ vector index. This object can be written in the compact form

$$
G_{A}=\frac{1}{2 P^{+}} P^{m}\left(\Gamma^{+} \Gamma_{m} D\right)_{A} .
$$

It will be useful to keep in mind the following $S O(9)$ relations which can be deduced from (3) and (4),

$$
\begin{aligned}
& \left\{D_{A}, D_{B}\right\}=-2 \sqrt{2} \delta_{A B} P^{-} \\
& \left\{\bar{D}_{A}, \bar{D}_{B}\right\}=-2 \sqrt{2} \delta_{A B} P^{+} \\
& \left\{D_{A}, \bar{D}_{B}\right\}=2\left[\left(\gamma^{9} \gamma^{\hat{i}}\right)_{A B} P_{\hat{i}}-\delta_{A B} P_{11}\right] \\
& \left\{\bar{D}_{A}, D_{B}\right\}=2\left[-\left(\gamma^{9} \gamma^{\hat{i}}\right)_{A B} P_{\hat{i}}-\delta_{A B} P_{11}\right],
\end{aligned}
$$

where $D_{A}$ and $\bar{D}_{A}$ are given by

$$
\begin{aligned}
& D_{A}=P_{A}+\sqrt{2} i \Theta_{A} P^{-}-i\left(\gamma^{9} \gamma^{\hat{i}} \bar{\Theta}\right)_{A} P_{\hat{i}}+i \bar{\Theta}_{A} P_{11} \\
& \bar{D}_{A}=\bar{P}_{A}+\sqrt{2} i \bar{\Theta}_{A} P^{+}+i\left(\gamma^{9} \gamma^{\hat{i}} \Theta\right)_{A} P_{\hat{i}}+i \Theta_{A} P_{11}
\end{aligned}
$$

or in a more compact form

$$
\begin{aligned}
& D_{A}=P_{A}+\sqrt{2} i \Theta_{A} P^{-}+\Gamma_{A B}^{I} \bar{\Theta}^{B} P_{I} \\
& \bar{D}_{A}=\bar{P}_{A}+\sqrt{2} i \bar{\Theta}_{A} P^{+}+\Gamma_{A B}^{I} \Theta^{B} P_{I},
\end{aligned}
$$

where $\Gamma_{A \bar{B}}^{I}=\left(-i\left(\gamma^{9} \gamma^{\hat{i}}\right)_{A B}, i \delta_{A B}\right), \Gamma_{\bar{A} B}^{I}=\left(i\left(\gamma^{9} \gamma^{\hat{i}}\right)_{A B}, i \delta_{A B}\right)$. Using Eqs. (57) and (58), one can show that

$$
\begin{gathered}
\left\{G_{A}, \bar{D}_{B}\right\}=0 \\
\left\{G_{A}, G_{B}\right\}=\frac{\sqrt{2}}{P^{+}}\left(P^{m} P_{m}\right) \delta_{A B} .
\end{gathered}
$$

Notice that the nilpotency of $\tilde{Q}$ no longer requires the validity of the $S O(9)$ pure spinor constraint $\Lambda^{A} \Gamma_{A B}^{I} \bar{\Lambda}^{B}=0$ as can be seen from (63). A further similarity transformation induced by the operator,

$$
\hat{R}=-\frac{1}{\sqrt{2} P^{+}}\left(\Theta^{A} \Gamma_{A B}^{I} \bar{P}^{B}\right) P_{I},
$$

will transform the operators $\bar{D}_{A}, G_{A}$ into

$$
\begin{gathered}
\hat{\bar{D}}_{A}=\bar{P}_{A}+\sqrt{2} i \bar{\Theta}^{A} P^{+} \\
\hat{G}_{A}=P_{A}-\frac{i}{\sqrt{2} P^{+}}\left(P^{m} P_{m}\right) \Theta_{A} .
\end{gathered}
$$

Hence, the pure spinor BRST operator will take the form

$$
\tilde{\tilde{Q}}=\Lambda^{A} \hat{G}_{A}+\bar{\Lambda}^{A} \hat{\bar{D}}_{A}
$$

The supersymmetry invariance of this operator follows from the supersymmetry invariance of $\hat{G}_{A}$ and $\hat{\bar{D}}_{A}$ under the operators

$$
\begin{gathered}
\hat{\bar{Q}}_{A}=\bar{P}_{A}-\sqrt{2} i \bar{\Theta}_{A} P^{+} \\
\hat{Q}_{A}=P_{A}+\frac{i}{\sqrt{2} P^{+}}\left(P^{m} P_{m}\right) \Theta_{A}-\frac{i}{\sqrt{2} P^{+}} P_{I} \Gamma_{A D}^{I} \hat{\bar{Q}}_{D},
\end{gathered}
$$

which are the $\tilde{R}$-transformed versions of the supersymmetry generators

$$
\begin{aligned}
& Q_{A}=P_{A}-\sqrt{2} i P^{-} \Theta_{A}+i\left(\gamma^{9} \gamma^{\hat{i}} \bar{\Theta}\right)_{A} P_{\hat{i}}-i \bar{\Theta}_{A} P_{11} \\
& \bar{Q}_{A}=\bar{P}_{A}-\sqrt{2} i P^{+} \bar{\Theta}_{A}-\left(\gamma^{9} \gamma^{\hat{i}} \Theta\right)_{A} P_{\hat{i}}-i \Theta_{A} P_{11} .
\end{aligned}
$$

\section{A. Light-cone equations of motion}

The physical fields are contained in the ghost-number 3 superfield $V=\Lambda^{\alpha} \Lambda^{\beta} \Lambda^{\sigma} C_{\alpha \beta \sigma}$ [5]. This superfield can be written in $S O(9)$ notation as

$$
\begin{aligned}
V= & \Lambda^{A} \Lambda^{B} \Lambda^{C} C_{(+A)(+B)(+C)}+3 \bar{\Lambda}^{A} \Lambda^{B} \Lambda^{C} C_{(-A)(+B)(+C)} \\
& +3 \bar{\Lambda}^{A} \bar{\Lambda}^{B} \Lambda^{C} C_{(-A)(-B)(+C)}+\bar{\Lambda}^{A} \bar{\Lambda}^{B} \bar{\Lambda}^{C} C_{(-A)(-B)(-C)},
\end{aligned}
$$

where the signs \pm come from the splitting $S O(10,1) \rightarrow S O(1,1) \times S O(9)$. The use of the gauge transformation $\delta V=\tilde{\tilde{Q}} \Omega$, with $\Omega$ being an arbitrary ghost-number 2 superfield, allows us to cancel out the last three terms in (73),

$$
\begin{aligned}
\tilde{\tilde{Q}} \Omega= & \Lambda^{A} \Lambda^{B} \Lambda^{C} \hat{G}_{A} \Omega_{(+B)(+C)}+2 \Lambda^{A} \bar{\Lambda}^{B} \Lambda^{C} \hat{G}_{A} \Omega_{(-B)(+C)} \\
& +\Lambda^{A} \bar{\Lambda}^{B} \bar{\Lambda}^{C} \hat{G}_{A} \Omega_{(-B)(-C)}+\bar{\Lambda}^{A} \Lambda^{B} \Lambda^{C} \hat{\bar{D}}_{A} \Omega_{(+B)(+C)} \\
& +2 \bar{\Lambda}^{A} \bar{\Lambda}^{B} \Lambda^{C} \hat{\bar{D}}_{A} \Omega_{(-B)(+C)}+\bar{\Lambda}^{A} \bar{\Lambda}^{B} \bar{\Lambda}^{C} \hat{\bar{D}}_{A} \Omega_{(-B)(-C)},
\end{aligned}
$$

after conveniently choosing $\Omega_{(-B)(-C)}, \quad \Omega_{(+B)(-C)}$, $\Omega_{(+B)(+C)}$. Therefore, we are left with

$$
V=\Lambda^{A} \Lambda^{B} \Lambda^{C} C_{A B C},
$$

where we have dropped the $S O(1,1)$ index for convenience. The $\tilde{\tilde{Q}}$-closedness condition for $V$ implies the following equations for $C_{B C D}$,

$$
\begin{aligned}
\hat{\bar{D}}_{A} C_{B C D} & =\left(\Gamma^{J}\right)_{A(B} C_{|J| C D)}+\delta_{(B C} \chi_{D) A} \\
\hat{G}_{A} C_{B C D}= & \delta_{(A B} \xi_{C D)}+\left(\Gamma^{J K}\right)_{A(B} C_{|J K| C D)} \\
& +\left(\Gamma^{J K L}\right)_{A(B} C_{|J K L| C D)},
\end{aligned}
$$


where $\chi_{D A}, \xi_{C D}, C_{J C D}, C_{J K C D}$, and $C_{J K L C D}$ are $S O(9)$ $p$-form bispinors. Each of these possesses a certain symmetry determined by (75) and (76). To find the physical spectrum and the corresponding equations of motion, we should solve these equations subject to the constraints

$$
\begin{gathered}
\left\{\hat{\bar{D}}_{A}, \hat{\bar{D}}_{B}\right\}=-2 \sqrt{2} P^{+} \delta_{A B} \\
\left\{\hat{G}_{A}, \hat{G}_{B}\right\}=\frac{\sqrt{2}}{P^{+}}\left(P^{m} P_{m}\right) \delta_{A B} .
\end{gathered}
$$

A way to solve this constrained system of equations is the following. Let us choose the only nonzero component of the spinor $\Lambda^{A}$ to be $\Lambda^{+0}$. This choice will imply $\bar{\Lambda}^{-i}=\bar{\Lambda}^{+0}=0$, where $i$ is the usual $S O(7)$ vector index. With these constraints, the only $\hat{\bar{D}}_{A}$ that act nontrivially on $C_{(+0)(+0)(+0)}$ are $\hat{\bar{D}}_{-i}$ and $\hat{\bar{D}}_{+0}$. Therefore, we will have $2^{8}$ states in $C_{(+0)(+0)(+0)}: 128$ bosonic and 128 fermionic states. The other components of $C_{A B C}$ can be shown to be related to $C_{(+0)(+0)(+0)}$ by $S O(9)$ rotations (see Appendix C) given by the operator

$$
R^{I J}=\frac{1}{\sqrt{8 \sqrt{2} P^{+}}}\left(\hat{\bar{D}} \Gamma^{I J} \hat{\bar{D}}\right)
$$

which satisfies the algebra

$$
\left[R^{I J}, R^{K L}\right]=\eta^{I K} R^{J L}-\eta^{J K} R^{I L}-\eta^{I L} R^{J K}+\eta^{J L} R^{I K} .
$$

The 128 fermionic states can be adequately represented by the lowest order term in $\tilde{f}_{J D}$,

$$
C_{B C D}=\left(\Gamma^{J}\right)_{(B C} \tilde{f}_{|J| D)},
$$

where $\tilde{f}_{J D}$ is $\Gamma$ traceless. The 128 bosonic states can be accommodated in the $S O(9)$ traceless symmetric tensor $g_{J K}$ and the 3-form $H^{L M N}$. Therefore, we can write

$$
C_{J C D}=a\left(\Gamma^{K}\right)_{C D} g_{J K}+b\left(\Gamma_{J K L M}\right)_{C D} H^{K L M} .
$$

After replacing (81) and (82) in (75), one obtains

$$
\begin{aligned}
\Gamma_{(B C}^{J} \bar{D}_{\mid A} \tilde{f}_{J \mid D)}= & a\left(\Gamma^{K}\right)_{A(B}\left(\Gamma^{J}\right)_{C D)} g_{J K} \\
& +b\left(\Gamma_{J}\right)_{A(B}\left(\Gamma^{J K L M}\right)_{C D)} H_{K L M} \\
& +\frac{2 b}{3} \delta_{(B C}\left(\Gamma^{K L M}\right)_{D) A} H_{K L M} .
\end{aligned}
$$

Next, we use the $S O(9)$ Fierz identities

$$
\begin{aligned}
\delta_{(B C}\left(\Gamma^{K L M}\right)_{D) A}= & 3\left(\Gamma^{[K}\right)_{(B C}\left(\Gamma^{L M]}\right)_{D) A} \\
& +\left(\Gamma_{J}\right)_{(B C}\left(\Gamma^{J K L M}\right)_{D) A} \\
\left(\Gamma^{J K L M}\right)_{(B C}\left(\Gamma_{J}\right)_{D) A} & =-\left(\Gamma_{J}\right)_{(B C}\left(\Gamma^{J K L M}\right)_{D) A},
\end{aligned}
$$

which can be found by using the Mathematica package GAMMA [12], to obtain

$$
\begin{aligned}
\Gamma_{(B C}^{J} \bar{D}_{\mid A} \tilde{f}_{J \mid D)}= & a\left(\Gamma^{J}\right)_{(B C}\left(\Gamma^{K}\right)_{D) A} g_{J K} \\
& +2 b\left(\Gamma^{J}\right)_{(B C}\left(\Gamma^{L M}\right)_{D) A} H_{J L M} \\
& -\frac{b}{3}\left(\Gamma_{J}\right)_{(B C}\left(\Gamma^{J K L M}\right)_{D) A} H_{K L M},
\end{aligned}
$$

which implies

$$
\begin{aligned}
\hat{\bar{D}}_{A} \tilde{f}_{J D}= & a\left(\Gamma^{K}\right)_{A D} g_{J K}-2 b\left(\Gamma^{L M}\right)_{A D} H_{J L M} \\
& -\frac{b}{3}\left(\Gamma_{J K L M}\right)_{A D} H^{K L M},
\end{aligned}
$$

where the constants $a$ and $b$ will be determined from supersymmetry. To do this, we should know how $\hat{\bar{D}}_{A}$ acts on $g_{J K}$ and $H_{K L M}$. An educated guess based on linearity and symmetry properties is

$$
\begin{aligned}
\hat{\bar{D}}_{A} g_{J K} & =-2 \sqrt{2} P^{+}\left[\left(\Gamma_{J}\right)_{A E} \tilde{f}_{K E}+\left(\Gamma_{K}\right)_{A E} \tilde{f}_{J E}\right] \\
\hat{\bar{D}}_{A} H^{K L M}= & -2 \sqrt{2} P^{+}\left[\left(\Gamma^{K L}\right)_{A E} \tilde{f}_{E}^{M}-\left(\Gamma^{K M}\right)_{A E} \tilde{f}_{E}^{L}\right. \\
& \left.+\left(\Gamma^{L M}\right)_{A E} \tilde{f}_{E}^{K}\right],
\end{aligned}
$$

where the factor $-2 \sqrt{2} P^{+}$was chosen for convenience. These equations of motion should satisfy the supersymmetry algebra (77). This requirement fixes the values of $a$ and $b$ to be $a=\frac{1}{4}$ and $b=\frac{1}{72}$. Therefore, the whole set of light-cone equations of motion is

$$
\begin{aligned}
\hat{\bar{D}}_{A} g_{J K}= & -2 \sqrt{2} P^{+}\left[\left(\Gamma_{J}\right)_{A E} \tilde{f}_{K E}+\left(\Gamma_{K}\right)_{A E} \tilde{f}_{J E}\right] \\
\hat{\bar{D}}_{A} \tilde{f}_{J D}= & \frac{1}{4}\left(\Gamma^{K}\right)_{A D} g_{J K}+\frac{1}{72}\left[\left(\Gamma_{J K L M}\right)_{A D}\right. \\
& \left.+6 \eta^{J K}\left(\Gamma^{L M}\right)_{A D}\right] H^{K L M} \\
\hat{\bar{D}}_{A} H^{K L M}= & -2 \sqrt{2} P^{+}\left[\left(\Gamma^{K L}\right)_{A E} \tilde{f}_{E}^{M}-\left(\Gamma^{K M}\right)_{A E} \tilde{f}_{E}^{L}\right. \\
+ & \left.\left(\Gamma^{L M}\right)_{A E} \tilde{f}_{E}^{K}\right] .
\end{aligned}
$$

These expressions are the same equations of motion obtained for $D=11$ linearized supergravity from the light-cone $D=11$ Brink-Schwarz-like superparticle [7].

The mass-shell condition can be obtained from (78) after using the tracelessness condition for $C_{B C D}$, which is necessary to have a nontrivial vertex operator $V$. This condition gives rise to the equation

$$
\hat{G}_{A} C_{B C D}+\hat{G}_{B} C_{A C D}+\hat{G}_{C} C_{A B D}+\hat{G}_{D} C_{A B C}=0,
$$

which has a solution only if $\hat{G}_{A} C_{B C D}=0$. This result, together with (78), implies that $k^{m} k_{m}=0$, where $k^{m}$ is the momentum. Consequently, $C_{B C D}$ depends only on $\bar{\Theta}$, $C_{B C D}=C_{B C D}(\bar{\Theta})$. To obtain the pure spinor vertex operator in the $Q$-cohomology, one just performs the similarity transformation generated by $-(R+\hat{R})$. The result is 


$$
V=V(\hat{\bar{\Theta}}) e^{i k \cdot X}
$$

where $\hat{\bar{\Theta}}^{A}=\bar{\Theta}^{A}-\frac{i}{\sqrt{2} P^{+}} \Theta_{B}\left(\Gamma^{I}\right)^{A B} k_{I}$.

\section{REMARKS}

The equivalence of cohomologies for the $D=11$ BrinkSchwarz-like superparticle and the $D=11$ pure spinor superparticle is strong evidence that the two models describe the same physical theory. Our method to demonstrate the equivalence uses ideas that were applied previously to the $D=10$ case [e.g., the group decomposition $S O(10,1) \rightarrow S O(1,1) \times S O(9)]$ and introduces a parametrization of $D=11$ objects [the group decomposition $S O(10,1) \rightarrow S O(3,1) \times S O(7)]$, which was useful for analyzing the light-cone pure spinor cohomology.

The equations of motion in $D=9$ superspace found in this paper, by studying the light-cone pure spinor cohomology, match the light-cone equations of motion presented in Ref. [7]. We conclude that the $D=11$ pure spinor superparticle is a good model to study $D=11$ linearized supergravity in a manifestly covariant way.

\section{ACKNOWLEDGMENTS}

I would like to thank Nathan Berkovits for very useful discussions and FAPESP for financial support from Grant No. $15 / 23732-2$.

\section{APPENDIX A: $\Gamma$-MATRICES OF $S O(10,1)$}

We will denote $S O(10,1)$ vector indices by $m, n, \ldots$ and $S O(9,1)$ vector indices by $\hat{m}, \hat{n}, \ldots$. In addition, we will denote $S O(10,1)$ spinor indices by $\alpha, \beta, \ldots$ and $S O(9,1)$ spinor indices by $\mu, \nu, \ldots$. As usual, we add a new matrix, $\Gamma^{10}$, to the set of $S O(9,1)$ gamma matrices $\left\{\Gamma^{\hat{m}}\right\}$, which is numerically equal to the chirality matrix $\Gamma^{(9,1)}$ in $D=(9,1)$ :

$$
\Gamma^{10}=\Gamma^{(9,1)}=\left(\begin{array}{cc}
I_{16 \times 16} & 0 \\
0 & -I_{16 \times 16}
\end{array}\right) .
$$

This matrix satisfies the properties $\left\{\Gamma^{m}, \Gamma^{10}\right\}=0$, for $m=0, \ldots, 9$, and $\left(\Gamma^{10}\right)^{2}=1$. The chirality matrix $\Gamma$ in $D=$ $(10,1)$ is given by

$$
\Gamma=\Gamma^{0} \Gamma^{1} \ldots \Gamma^{9} \Gamma^{10}=\Gamma^{(9,1)} \Gamma^{10}=\left(\Gamma^{10}\right)^{2}=1,
$$

which reflects the fact that we do not have Weyl (anti-Weyl) spinors in this case. However, we can have Majorana spinors. It is easy to see that $C=\Gamma^{0}$ satisfies the definition of the charge conjugation matrix ${ }^{5} C \Gamma^{m}=-\left(\Gamma^{m}\right)^{T} C$. For two Majorana spinors $\Theta$ and $\Psi$, we have $\bar{\Theta} \Gamma^{m} \Psi=\Theta^{T} C \Gamma^{m} \Psi$. This result can be viewed in terms of $S O(9,1)$ components,

\footnotetext{
${ }^{5}$ We know that for $D=(9,1), C^{(9,1)}=\Gamma^{0}$ is the charge conjugation matrix, so we just need to show that $C=\Gamma^{0}$ obeys $C \Gamma^{10}=-\left(\Gamma^{10}\right)^{T} C$, which is trivial since $\Gamma^{10}$ is symmetric and $\left\{\Gamma^{10}, \Gamma^{0}\right\}=0$.
}

$\Theta^{T} C \Gamma^{m} \Psi=\left(\begin{array}{ll}\Theta^{\mu} & \Theta_{\mu}\end{array}\right)\left(\begin{array}{cc}\gamma_{\mu \nu}^{\hat{m}} & 0 \\ 0 & -\left(\gamma^{\hat{m}}\right)^{\mu \nu}\end{array}\right)\left(\begin{array}{ll}\Psi^{\nu} & \Psi_{\nu}\end{array}\right)$,

$\Theta^{T} C \Gamma^{10} \Psi=\left(\begin{array}{ll}\Theta^{\mu} & \Theta_{\mu}\end{array}\right)\left(\begin{array}{cc}0 & -1 \\ -1 & 0\end{array}\right)\left(\begin{array}{ll}\Psi^{\nu} & \Psi_{\nu}\end{array}\right)$,

where $m=0, \ldots, 9$ and $\gamma_{\mu \nu}^{\hat{m}}$, and $\left(\gamma^{\hat{m}}\right)^{\mu \nu}$ are the $S O(9,1)$ $\gamma$-matrices. It is useful to mention that the index structure of the charge conjugation matrix is $C_{\alpha \beta}$. So, the $\Gamma$-matrices have index structure $\left(\Gamma^{m}\right)^{\alpha}{ }_{\beta}$, and when they are multiplied by the charge conjugation matrix (or its inverse), we obtain the corresponding matrices $\left(\Gamma^{m}\right)_{\alpha \beta}$ and $\left(\Gamma^{m}\right)^{\alpha \beta}$.

Next, we will show explicitly the form of the gamma matrices. For $D=(9,1)$, we have

$$
\begin{aligned}
& \left(\gamma^{0}\right)^{\alpha \beta}=\left(\begin{array}{cc}
1_{8 \times 8} & 0 \\
0 & 1_{8 \times 8}
\end{array}\right), \quad\left(\gamma^{0}\right)_{\alpha \beta}=\left(\begin{array}{cc}
-1_{8 \times 8} & 0 \\
0 & -1_{8 \times 8}
\end{array}\right) \\
& \left(\gamma^{9}\right)^{\alpha \beta}=\left(\begin{array}{cc}
1_{8 \times 8} & 0 \\
0 & -1_{8 \times 8}
\end{array}\right), \quad\left(\gamma^{9}\right)_{\alpha \beta}=\left(\begin{array}{cc}
1_{8 \times 8} & 0 \\
0 & -1_{8 \times 8}
\end{array}\right) \\
& \left(\gamma^{\hat{i}}\right)^{\alpha \beta}=\left(\begin{array}{cc}
0 & \sigma_{a \dot{\hat{i}}}^{\hat{i}} \\
\sigma_{\dot{b} b}^{\hat{i}} & 0
\end{array}\right), \quad\left(\gamma^{\hat{i}}\right)_{\alpha \beta}=\left(\begin{array}{cc}
0 & \sigma_{a \dot{\hat{i}}}^{\hat{i}} \\
\sigma_{\dot{b} b}^{\hat{i}} & 0
\end{array}\right) \\
& \left(\gamma^{+}\right)^{\alpha \beta}=\left(\begin{array}{cc}
\sqrt{2}_{8 \times 8} & 0 \\
0 & 0
\end{array}\right), \quad\left(\gamma^{+}\right)_{\alpha \beta}=\left(\begin{array}{cc}
0 & 0 \\
0 & -\sqrt{2}_{8 \times 8}
\end{array}\right) \\
& \left(\gamma^{-}\right)^{\alpha \beta}=\left(\begin{array}{cc}
0 & 0 \\
0 & \sqrt{2}_{8 \times 8}
\end{array}\right), \quad\left(\gamma^{-}\right)_{\alpha \beta}=\left(\begin{array}{cc}
-\sqrt{2}_{8 \times 8} & 0 \\
0 & 0
\end{array}\right),
\end{aligned}
$$

where each entry is an $8 \times 8$ matrix and $\hat{i}$ is a $S O(8)$ vector index. The matrices $\gamma^{ \pm}$are defined by

$$
\gamma^{ \pm}=\frac{1}{\sqrt{2}}\left(\gamma^{0} \pm \gamma^{9}\right)
$$

The $\sigma^{\hat{i}}$-matrices are defined by

$$
\begin{array}{ll}
\sigma_{a \dot{a}}^{1}=\epsilon \otimes \epsilon \otimes \epsilon & \sigma_{a \dot{a}}^{5}=\tau^{3} \otimes \epsilon \otimes 1 \\
\sigma_{a \dot{a}}^{2}=1 \otimes \tau^{1} \otimes \epsilon & \sigma_{a \dot{a}}^{6}=\epsilon \otimes 1 \otimes \tau^{1} \\
\sigma_{a \dot{a}}^{3}=1 \otimes \tau^{3} \otimes \epsilon & \sigma_{a \dot{a}}^{7}=\epsilon \otimes 1 \otimes \tau^{3} \\
\sigma_{a \dot{a}}^{4}=\tau^{1} \otimes \epsilon \otimes 1 & \sigma_{a \dot{a}}^{8}=1 \otimes 1 \otimes 1,
\end{array}
$$

where $\epsilon=i \tau^{2}$ and $\tau^{1}, \tau^{2}$, and $\tau^{3}$ are the usual Pauli matrices. The $\sigma_{\dot{a} a}^{\hat{i}}$ are symmetric $\left(\sigma_{\dot{a} a}^{\hat{i}}=\left(\sigma_{a \dot{a}}^{\hat{i}}\right)^{T}\right)$ and satisfy the following relations: 


$$
\sigma_{a \dot{a}}^{\hat{i}} \sigma_{\dot{a} b}^{\hat{j}}+\sigma_{a \dot{a}}^{\hat{j}} \sigma_{\dot{a} b}^{\hat{i}}=2 \delta^{\hat{\hat{j}} \hat{j}} \delta_{a b} \quad \sigma_{\dot{a} a}^{\hat{i}} \sigma_{a \dot{b}}^{\hat{j}}+\sigma_{\dot{a} a}^{\hat{j}} \sigma_{a \dot{b}}^{\hat{i}}=2 \delta^{\hat{\hat{i}} \hat{j}} \delta_{\dot{a} \dot{b}} \quad \sigma_{\dot{a} b}^{\hat{i}} \sigma_{a \dot{c}}^{\hat{i}}+\sigma_{\dot{a} a}^{\hat{i}} \sigma_{b \dot{c}}^{\hat{i}}=2 \delta_{a b} \delta_{\dot{a} \dot{c}} .
$$

Similarly, for $D=(10,1)$, we have

$$
\begin{aligned}
& \left(\Gamma^{\hat{i}}\right)^{\alpha \beta}=\left(\begin{array}{cc}
-i \gamma^{\hat{i} A B} & O \\
O & i \gamma_{A B}^{\hat{i}}
\end{array}\right), \quad\left(\Gamma^{\hat{i}}\right)_{\alpha \beta}=\left(\begin{array}{cc}
i \gamma_{A B}^{\hat{i}} & O \\
O & -i \gamma^{\hat{i} A B}
\end{array}\right) \\
& \left(\Gamma^{11}\right)^{\alpha \beta}=\left(\begin{array}{cc}
O & -i \\
-i & O
\end{array}\right), \quad\left(\Gamma^{11}\right)_{\alpha \beta}=\left(\begin{array}{cc}
O & i \\
i & O
\end{array}\right) \\
& \left(\Gamma^{+}\right)^{\alpha \beta}=\left(\begin{array}{cc}
\left(\begin{array}{cc}
-\sqrt{2} i & 0 \\
0 & 0
\end{array}\right) & O \\
O & \left(\begin{array}{cc}
0 & 0 \\
0 & -\sqrt{2} i
\end{array}\right)
\end{array}\right), \quad\left(\Gamma^{+}\right)_{\alpha \beta}=\left(\begin{array}{cc}
\left(\begin{array}{cc}
0 & 0 \\
0 & -\sqrt{2} i
\end{array}\right) & O \\
O & \left(\begin{array}{cc}
-\sqrt{2} i & 0 \\
0 & 0
\end{array}\right)
\end{array}\right) \\
& \left(\Gamma^{-}\right)^{\alpha \beta}=\left(\begin{array}{ccc}
\left(\begin{array}{cc}
0 & 0 \\
0 & -\sqrt{2} i
\end{array}\right) & O & \\
O & \left(\begin{array}{cc}
-\sqrt{2} i & 0 \\
0 & 0
\end{array}\right)
\end{array}\right), \quad\left(\Gamma^{-}\right)_{\alpha \beta}=\left(\begin{array}{cc}
\left(\begin{array}{cc}
-\sqrt{2} i & 0 \\
0 & 0
\end{array}\right) & O \\
O & \left(\begin{array}{cc}
0 & 0 \\
0 & -\sqrt{2} i
\end{array}\right)
\end{array}\right) \text {, }
\end{aligned}
$$

where $A$ and $B$ are $S O(9)$ spinor indices. Notice that each $\Gamma$-matrix is $32 \times 32$.

To construct the above representation of the $\Gamma$-matrices, we used a basis convenient for dealing with $S O(8)$ objects. Hence, an arbitrary $D=11$ spinor $\chi^{\alpha}$ is written in this basis as

$$
\chi^{\alpha}=\left(\begin{array}{c}
\chi^{a} \\
\chi^{\dot{a}} \\
\bar{\chi}^{a} \\
\bar{\chi}^{\dot{a}}
\end{array}\right) .
$$

This was the convention used in (26). This is useful when $S O(8)$ objects are needed, as in Sec. III. However, when analyzing the light-cone structure of the pure spinor cohomology and vertex operators, we need to deal with $S O(9)$ objects. So, we define the following change of basis matrix,

$$
M_{c b m}=\left(\begin{array}{cccc}
0 & 0 & 1 & 0 \\
0 & 1 & 0 & 0 \\
1 & 0 & 0 & 0 \\
0 & 0 & 0 & 1
\end{array}\right),
$$

where each entry represents an $8 \times 8$ matrix. Using this matrix, we find the corresponding $\Gamma$-matrices in this new basis,

$$
\begin{array}{rlrl}
\left(\Gamma^{\hat{i}}\right)^{\alpha \beta} & =i\left(\begin{array}{cc}
O & \left(\gamma^{9} \gamma^{\hat{i}}\right)^{A B} \\
-\left(\gamma^{9} \gamma^{\hat{i}}\right)_{A B} & O
\end{array}\right), \\
\left(\Gamma^{\hat{i}}\right)_{\alpha \beta} & =i\left(\begin{array}{cc}
O & -\left(\gamma^{9} \gamma^{\hat{i}}\right)^{A B} \\
\left(\gamma^{9} \gamma^{\hat{i}}\right)_{A B} & O
\end{array}\right) \\
\left(\Gamma^{11}\right)^{\alpha \beta} & =-i\left(\begin{array}{cc}
O & I^{A B} \\
I_{A B} & O
\end{array}\right), & \left(\Gamma^{11}\right)_{\alpha \beta}=i\left(\begin{array}{cc}
O & I_{A B} \\
I^{A B} & O
\end{array}\right) \\
\left(\Gamma^{+}\right)^{\alpha \beta} & =\left(\begin{array}{cc}
O & O \\
O & -\sqrt{2} i
\end{array}\right), & \left(\Gamma^{+}\right)_{\alpha \beta}=\left(\begin{array}{cc}
-\sqrt{2} i & O \\
O & O
\end{array}\right) \\
\left(\Gamma^{-}\right)^{\alpha \beta} & =\left(\begin{array}{cc}
-\sqrt{2} i & O \\
O & O
\end{array}\right), & \left(\Gamma^{-}\right)_{\alpha \beta}=\left(\begin{array}{cc}
O & O \\
O & -\sqrt{2} i
\end{array}\right),
\end{array}
$$

where $I_{A B}$ is the $S O(9)$ identity matrix, $A$ and $B$ are $S O(9)$ spinor indices, and $\hat{i}=1, \ldots, 8$. Each entry in the above matrices is $16 \times 16$.

\section{APPENDIX B: $S O(10,1) \rightarrow S O(3,1) \times S O(7)$}

Here, we will explain the \pm notation and construct explicitly a different representation for the $S O(10,1)$ gamma matrices. Let us define the raising and lowering $\Gamma$-matrices:

$$
\begin{aligned}
\Gamma^{ \pm 0+1} & =\frac{1}{2}\left( \pm \Gamma^{0}+\Gamma^{1}\right) \\
\Gamma^{2 \pm 3 i} & =\frac{1}{2}\left(\Gamma^{2} \pm i \Gamma^{3}\right) .
\end{aligned}
$$


These $\Gamma^{ \pm}$-matrices act on an arbitrary spinor $\chi$ as follows:

$$
\begin{array}{rlrlrl}
\Gamma^{0+1}|-+a\rangle & =|++a\rangle, & & \Gamma^{3+4 i}|--a\rangle=-|-+a\rangle, & & \Gamma^{j}|++0\rangle=|++j\rangle \\
\Gamma^{0+1}|--a\rangle & =|+-a\rangle, & & \Gamma^{3+4 i}|+-a\rangle=|++a\rangle, & & \Gamma^{j}|++j\rangle=|++0\rangle \\
\Gamma^{-0+1}|++a\rangle & =|-+a\rangle, & & \Gamma^{3-4 i}|-+a\rangle=-|-+a\rangle, & & \Gamma^{j}|-+0\rangle=-|-+j\rangle \\
\Gamma^{-0+1}|+-a\rangle & =|--a\rangle, & & \Gamma^{3-4 i}|++a\rangle=|+-a\rangle, & & \Gamma^{j}|-+j\rangle=-|-+0\rangle \\
\Gamma^{j}|--0\rangle & =|--j\rangle, & & \Gamma^{j}|--j\rangle=|--0\rangle, & \Gamma^{j}|+-0\rangle=-|+-j\rangle \\
\Gamma^{j}|+-j\rangle & =-|+-0\rangle, & &
\end{array}
$$

and any other relation vanishes. In these formulas, we have made the identification $| \pm \pm a\rangle=\chi^{ \pm \pm a}$ with $a=0, i$. It is clear that these relations are consistent with the $S O(10,1)$ Clifford algebra. With these rules, one can construct the respective representation

$$
\left(\Gamma^{0+1}\right)_{\beta}^{\alpha}=\left(\begin{array}{cccc}
0 & 0 & 0 & 1 \\
0 & 0 & 0 & 0 \\
0 & 1 & 0 & 0 \\
0 & 0 & 0 & 0
\end{array}\right), \quad\left(\Gamma^{-0+1}\right)_{\beta}^{\alpha}=\left(\begin{array}{cccc}
0 & 0 & 0 & 0 \\
0 & 0 & 1 & 0 \\
0 & 0 & 0 & 0 \\
1 & 0 & 0 & 0
\end{array}\right)
$$

Here and throughout this Appendix, each entry will represent an $8 \times 8$ matrix unless otherwise stated. Now, it is easy to calculate the explicit form of the matrices $\left(\Gamma^{0}\right)_{\beta}^{\alpha}$ and $\left(\Gamma^{1}\right)_{\beta}^{\alpha}$ :

$$
\left(\Gamma^{0}\right)_{\beta}^{\alpha}=\left(\begin{array}{cccc}
0 & 0 & 0 & 1 \\
0 & 0 & -1 & 0 \\
0 & 1 & 0 & 0 \\
-1 & 0 & 0 & 0
\end{array}\right), \quad\left(\Gamma^{1}\right)_{\beta}^{\alpha}=\left(\begin{array}{cccc}
0 & 0 & 0 & 1 \\
0 & 0 & 1 & 0 \\
0 & 1 & 0 & 0 \\
1 & 0 & 0 & 0
\end{array}\right) .
$$

Similarly, we find

$$
\left(\Gamma^{2+3 i}\right)_{\beta}^{\alpha}=\left(\begin{array}{cccc}
0 & 0 & 1 & 0 \\
0 & 0 & 0 & 0 \\
0 & 0 & 0 & 0 \\
0 & -1 & 0 & 0
\end{array}\right) \quad\left(\Gamma^{2-3 i}\right)_{\beta}^{\alpha}=\left(\begin{array}{cccc}
0 & 0 & 0 & 0 \\
0 & 0 & 0 & -1 \\
1 & 0 & 0 & 0 \\
0 & 0 & 0 & 0
\end{array}\right) \quad\left(\Gamma^{2}\right)_{\beta}^{\alpha}=\left(\begin{array}{cccc}
0 & 0 & 1 & 0 \\
0 & 0 & 0 & -1 \\
1 & 0 & 0 & 0 \\
0 & -1 & 0 & 0
\end{array}\right) \quad\left(\Gamma^{3}\right)_{\beta}^{\alpha}=\left(\begin{array}{cccc}
0 & 0 & -i & 0 \\
0 & 0 & 0 & -i \\
i & 0 & 0 & 0 \\
0 & i & 0 & 0
\end{array}\right) .
$$

However, as already mentioned, there exists an antisymmetric metric tensor $C_{\alpha \beta}$ in $D=11$ dimensions which raises and lowers indices. Let us define it as follows,

$$
C_{\alpha \beta}=\left(\begin{array}{cccc}
0 & -B & 0 & 0 \\
B & 0 & 0 & 0 \\
0 & 0 & 0 & -B \\
0 & 0 & B & 0
\end{array}\right), \quad\left(C^{-1}\right)^{\alpha \beta}=\left(\begin{array}{cccc}
0 & B & 0 & 0 \\
-B & 0 & 0 & 0 \\
0 & 0 & 0 & B \\
0 & 0 & -B & 0
\end{array}\right),
$$

where $B$ is a diagonal matrix with elements $B_{00}=1, B_{j j}=-1$. To preserve the original Clifford algebra, we need to multiply the matrices $\left(\Gamma^{a}\right)^{\alpha}{ }_{\beta}$ by $i$. Now, we can find the matrices $\left(\Gamma^{m}\right)_{\alpha \beta},\left(\Gamma^{m}\right)^{\alpha \beta}$ :

$$
\Gamma_{\alpha \beta}^{-0+1}=\left(\begin{array}{cccc}
0 & 0 & -i B & 0 \\
0 & 0 & 0 & 0 \\
-i B & 0 & 0 & 0 \\
0 & 0 & 0 & 0
\end{array}\right), \quad \Gamma_{\alpha \beta}^{0+1}=\left(\begin{array}{cccc}
0 & 0 & 0 & 0 \\
0 & 0 & 0 & i B \\
0 & 0 & 0 & 0 \\
0 & i B & 0 & 0
\end{array}\right) .
$$


and so

$$
\Gamma_{\alpha \beta}^{0}=\left(\begin{array}{cccc}
0 & 0 & i B & 0 \\
0 & 0 & 0 & i B \\
i B & 0 & 0 & 0 \\
0 & i B & 0 & 0
\end{array}\right), \quad \Gamma_{\alpha \beta}^{1}=\left(\begin{array}{cccc}
0 & 0 & -i B & 0 \\
0 & 0 & 0 & i B \\
-i B & 0 & 0 & , 0 \\
0 & i B & 0 & 0
\end{array}\right)
$$

By using $\left(\Gamma^{m}\right)^{\alpha \beta}=C^{\alpha \delta} C^{\beta \lambda}\left(\Gamma^{m}\right)_{\delta \lambda}$, we find the matrices $\Gamma^{0 \alpha \beta}$ and $\Gamma^{1 \alpha \beta}$ :

$$
\Gamma^{0 \alpha \beta}=\left(\begin{array}{cccc}
0 & B & 0 & 0 \\
-B & 0 & 0 & 0 \\
0 & 0 & 0 & B \\
0 & 0 & -B & 0
\end{array}\right)\left(\begin{array}{cccc}
0 & 0 & i B & 0 \\
0 & 0 & 0 & i B \\
i B & 0 & 0 & 0 \\
0 & i B & 0 & 0
\end{array}\right)\left(\begin{array}{cccc}
0 & -B & 0 & 0 \\
B & 0 & 0 & 0 \\
0 & 0 & 0 & -B \\
0 & 0 & B & 0
\end{array}\right)=\left(\begin{array}{cccc}
0 & 0 & i B & 0 \\
0 & 0 & 0 & i B \\
i B & 0 & 0 & 0 \\
0 & i B & 0 & 0
\end{array}\right)
$$

and

$$
\Gamma^{1 \alpha \beta}=\left(\begin{array}{cccc}
0 & 0 & i B & 0 \\
0 & 0 & 0 & -i B \\
i B & 0 & 0 & 0 \\
0 & -i B & 0 & 0
\end{array}\right)
$$

Analogously, we can find the remaining matrices

$$
\begin{aligned}
& \left(\Gamma^{2+3 i}\right)_{\alpha \beta}=\left(\begin{array}{cccc}
0 & 0 & 0 & 0 \\
0 & 0 & i B & 0 \\
0 & i B & 0 & 0 \\
0 & 0 & 0 & 0
\end{array}\right), \quad\left(\Gamma^{2-3 i}\right)_{\alpha \beta}=\left(\begin{array}{cccc}
0 & 0 & 0 & i B \\
0 & 0 & 0 & 0 \\
0 & 0 & 0 & 0 \\
i B & 0 & 0 & 0
\end{array}\right) \\
& \left(\Gamma^{2}\right)_{\alpha \beta}=\left(\begin{array}{cccc}
0 & 0 & 0 & i B \\
0 & 0 & i B & 0 \\
0 & i B & 0 & 0 \\
i B & 0 & 0 & 0
\end{array}\right), \quad\left(\Gamma^{3}\right)_{\alpha \beta}=\left(\begin{array}{cccc}
0 & 0 & 0 & -B \\
0 & 0 & B & 0 \\
0 & B & 0 & 0 \\
-B & 0 & 0 & 0
\end{array}\right) \\
& \left(\Gamma^{2}\right)^{\alpha \beta}=\left(\begin{array}{cccc}
0 & 0 & 0 & -i B \\
0 & 0 & -i B & 0 \\
0 & -i B & 0 & 0 \\
-i B & 0 & 0 & 0
\end{array}\right), \quad\left(\Gamma^{3}\right)^{\alpha \beta}=\left(\begin{array}{cccc}
0 & 0 & 0 & -B \\
0 & 0 & B & 0 \\
0 & B & 0 & 0 \\
-B & 0 & 0 & 0
\end{array}\right) \\
& \left(\Gamma^{i}\right)_{\alpha \beta}=\left(\begin{array}{cccc}
0 & -i B A & 0 & 0 \\
i B A & 0 & 0 & 0 \\
0 & 0 & 0 & i B A \\
0 & 0 & -i B A & 0
\end{array}\right), \quad\left(\Gamma^{i}\right)^{\alpha \beta}=\left(\begin{array}{cccc}
0 & i B A & 0 & 0 \\
-i B A & 0 & 0 & 0 \\
0 & 0 & 0 & -i B A \\
0 & 0 & i B A & 0
\end{array}\right) \text {, }
\end{aligned}
$$

where $A$ is an $8 \times 8$ matrix with nonvanishing elements $A_{0 j}=A_{j 0}=1$. All these matrices are symmetric and satisfy the desired property: $\Gamma_{\alpha \beta}^{m} \Gamma^{n \beta \lambda}+\Gamma_{\alpha \beta}^{n} \Gamma^{m \beta \lambda}=2 \eta^{m n} \delta_{\alpha}^{\lambda}$. 
Finally, the product of two spinors $\chi^{\alpha} \rho_{\alpha}$ will be defined as follows:

$$
\begin{aligned}
\chi^{\alpha} C_{\alpha \beta} \rho^{\beta}= & -\chi^{++0} \rho^{--0}+\chi^{++i} \rho^{--i}+\chi^{--0} \rho^{++0} \\
& -\chi^{--i} \rho^{++i}-\chi^{+-0} \rho^{-+0}+\chi^{+-i} \rho^{-+i} \\
& +\chi^{-+0} \rho^{+-0}-\chi^{-+i} \rho^{+-i} .
\end{aligned}
$$

\section{APPENDIX C: OCTONIONS AND $S O(7)$ ROTATIONS}

In this Appendix, we will show that any component of $C_{B C D}$ can be obtained from $C_{(+0)(+0)(+0)}$ by $S O(9)$ rotations. These rotations are defined by the operator

$$
R^{I J}=\frac{1}{\sqrt{8 \sqrt{2} P^{+}}} \hat{\bar{D}} \Gamma^{I J} \hat{\bar{D}}
$$

which satisfy the algebra

$$
\left[R^{I J}, R^{K L}\right]=\eta^{I K} R^{J L}-\eta^{J K} R^{I L}-\eta^{I L} R^{J K}+\eta^{J L} R^{I K} .
$$

Therefore, we can use this operator to rotate the ground state $C_{(+0)(+0)(+0)}$. To do this, let us first write the transformation rule for a general $C_{B C D}$ being acted on by $R^{I J}$ :

$$
\begin{aligned}
R^{I J} C_{B C D}= & \frac{1}{\sqrt{2}}\left(\Gamma^{I J}\right)_{B}{ }^{E} C_{E C D}+\frac{1}{\sqrt{2}}\left(\Gamma^{I J}\right)_{C}{ }^{E} C_{B E D} \\
& +\frac{1}{\sqrt{2}}\left(\Gamma^{I J}\right)_{D}{ }^{E} C_{B C E} .
\end{aligned}
$$

As explained above, only $\hat{\bar{D}}_{-i}$ and $\hat{\bar{D}}_{+0}$ will act nontrivially on $C_{(+0)(+0)(+0)}$. Thus, we have

$\left(\Gamma^{i j}\right)_{(+k)(-0)} \hat{\bar{D}}_{-k} \hat{\bar{D}}_{+0} C_{(+0)(+0)(+0)} \propto\left(\Gamma^{i j}\right)_{(+0)}{ }^{E} C_{E(+0)(+0)}$.

To solve this equation, we recall the notion of octonions [13].

The octonion mutiplication table can be written in the form

$$
e_{i} e_{j}=-\delta_{i j}+\epsilon_{i j k} e_{k},
$$

which is equivalent to

$$
e_{i} e_{j}=\delta_{i j}-i \epsilon_{i j k} e_{k},
$$

where $\epsilon_{i j k}$ is a totally antisymmetric tensor with value +1 when $(i j k)=(123),(145),(176),(246)$, (257), (347),
TABLE I. States produced by the rotation operator $R^{I J}$.

\begin{tabular}{lcc}
\hline \hline Initial state & $\begin{array}{c}\text { States produced } \\
\text { by } R^{i j}\end{array}$ & $\begin{array}{c}\text { States produced } \\
\text { by } R^{-k}\end{array}$ \\
\hline$C_{(+0)(+0)(+0)}$ & $C_{(+k)(+0)(+0)}$ & $C_{(-k)(+0)(+0)}$ \\
$C_{(+k)(+0)(+0)}$ & $C_{(+k)(+l)(+0)}$ & $C_{(-0)(+0)(+0)}, C_{(+l)(-j)(+0)}$ \\
$C_{(+k)(+l)(+0)}$ & $C_{(+k)(+l)(+r)}$ & $C_{(+k)(-0)(+0)}, C_{(+k)(+l)(-r)}$ \\
$C_{(-0)(+0)(+0)}$ & $\cdots$ & $C_{(-0)(-k)(+0)}$ \\
$C_{(+l)(-k)(+0)}$ & $C_{(-j)(-r)(+0)}$ & $C_{(+l)(-r)(-k)}$ \\
$C_{(+l)(+k)(+r)}$ & $\cdots$ & $C_{(-0)(+l)(+r)}$ \\
$C_{(+k)(-0)(+0)}$ & $\ldots$ & $C_{(-0)(-0)(+0)}, C_{(+k)(-0)(-r)}$ \\
$C_{(-0)(-k)(+0)}$ & $\ldots$ & $C_{(-0)(-k)(-r)}$ \\
$C_{(-k)(-r)(+0)}$ & $\cdots$ & $C_{(-k)(-r)(-t)}$ \\
$C_{(-0)(+l)(+r)}$ & $\cdots$ & $C_{(-0)(-0)(+r)}$ \\
$C_{(-0)(-0)(+0)}$ & $\cdots$ & $C_{(-0)(-0)(-r)}$ \\
$C_{(-0)(-0)(+r)}$ & $\cdots$ & $C_{(-0)(-0)(-0)}$ \\
\hline \hline
\end{tabular}

(365). Now, we can identify these octonions as the gamma matrices of the $S O(7)$ Clifford algebra:

$$
\Gamma^{i} \Gamma^{j}=\delta^{i j}-i \epsilon^{i j k} \Gamma^{k} .
$$

This equation can be thought of as the seven-dimensional generalization of the three-dimensional case

$$
\tau^{i} \tau^{j}=\delta^{i j}+i e^{i j k} \tau^{k}
$$

where $\tau^{i}$ are the ordinary Pauli matrices.

Coming back to Eq. (C4) and applying the octonion identity, we obtain

$$
\begin{aligned}
\left(\Gamma^{i j}\right)_{(+k)(-0)} \hat{\bar{D}}_{-k} \hat{\bar{D}}_{+0} C_{(+0)(+0)(+0)} & \propto\left(\Gamma^{i j}\right)_{(+0)} C_{E(+0)(+0)} \\
\epsilon^{i j k} \hat{\bar{D}}_{-k} \hat{\bar{D}}_{+0} C_{(+0)(+0)(+0)} & \propto \epsilon^{i j k} C_{(+k)(+0)(+0)} .
\end{aligned}
$$

Therefore, we have obtained the state $C_{(+i)(+0)(+0)}$. By acting with $R^{-k}$ on $C_{(+0)(+0)(+0)}$, we obtain the state $C_{(-i)(+0)(+0)}$ :

$$
\begin{aligned}
\left(\Gamma^{-k}\right)_{(+i)(+j)} \hat{\bar{D}}_{-i} \hat{\bar{D}}_{-j} C_{(+0)(+0)(+0)} & \propto\left(\Gamma^{-k}\right)_{(+0)}{ }^{E} C_{E(+0)(+0)} \\
\epsilon^{k i j} \hat{\bar{D}}_{-i} \hat{\bar{D}}_{-j} C_{(+0)(+0)(+0)} & \propto \delta^{k l} C_{(-l)(+0)(+0)} .
\end{aligned}
$$

In this way, one can obtain all states contained in $C_{A B C}$. The Table I shows explicitly how this is done. For brevity, we include only one way to obtain each state. The centered points $(\cdots)$ means that all states corresponding to an initial state have been already obtained from other initial states. Finally, since $C_{A B C}$ is completely symmetric, states related by symmetry to states on the table need not be included. 
[1] L. Brink and J. H. Schwarz, Phys. Lett. 100B, 310 (1981).

[2] N. Berkovits, J. High Energy Phys. 09 (2001) 016.

[3] O. A. Bedoya and N. Berkovits, in New Perspectives in String Theory Workshop Arcetri, Florence, Italy, 2009 (unpublished).

[4] N. Berkovits, in Superstrings and related matters. Proceedings, Spring School, Trieste, Italy, 2002 (2002), p. 57 [arXiv:hep-th/0209059].

[5] N. Berkovits, J. High Energy Phys. 09 (2002) 051.

[6] N. Berkovits and R. Lipinski Jusinskas, J. High Energy Phys. 08 (2014) 102.
[7] M. B. Green, M. Gutperle, and H. H. Kwon, J. High Energy Phys. 08 (1999) 012.

[8] L. Anguelova, P. A. Grassi, and P. Vanhove, Nucl. Phys. B702, 269 (2004).

[9] I. A. Bandos, Phys. Lett. B 659, 388 (2008).

[10] I. A. Bandos, Nucl. Phys. B796, 360 (2008).

[11] T. Kugo and I. Ojima, Prog. Theor. Phys. Suppl. 66, 1 (1979).

[12] U. Gran, arXiv:hep-th/0105086.

[13] J. C. Baez, Bull. Am. Math. Soc. 39, 145 (2002). 